\title{
1 Roles of Arabidopsis TBL34 and TBL35 in xylan acetylation and
}

2 plant growth

3

4 Youxi Yuan ${ }^{\mathrm{a}}$, Quincy Teng ${ }^{\mathrm{b}}$, Ruiqin Zhong ${ }^{\mathrm{a}}$ and Zheng-Hua Ye ${ }^{\mathrm{a}, *}$

5

6

7

$9{ }^{\mathrm{b}}$ Department of Pharmaceutical and Biomedical Sciences, University of Georgia, Athens, GA

1030602, USA

11

12

13

14

15

16

17

18

19

20

21

22

23

24

25

26

27

28

29

30

31

32

33

\section{* Correspondence:}

Zheng-Hua Ye

Department of Plant Biology

University of Georgia

Athens, GA 30602

USA

Tel: 17065421832

E-mail: yh@uga.edu 


\section{ABSTRACT}

3 xylosyl residues are acetylated at $O-2$ and/or $O-3$. Because acetylation of cell wall polymers

4 contributes to biomass recalcitrance for biofuel production, it is important to investigate the

5 biochemical mechanism underlying xylan acetylation, the knowledge of which could be applied

6 to custom-design biomass composition tailored for biofuel production. In this report, we

7 investigated the functions of Arabidopsis TRICHOME BIREFRINGENCE-LIKE 34 (TBL34)

8 and TBL35, two DUF231-containing proteins, in xylan acetylation. The TBL34 gene was found

9 to be specifically expressed in xylem cells in stems and root-hypocotyls, and both TBL34 and

10 TBL35 were shown to be localized in the Golgi, where xylan biosynthesis occurs. Chemical

11 analysis revealed that simultaneous mutations of TBL34 and TBL35 caused a mild decrease in

12 xylan acetyl content and a specific reduction in xylan 3-O-monoacetylation and 2,3-di-O-

13 acetylation. Furthermore, simultaneous mutations of TBL34, TBL35 and ESKIMO1 (ESK1)

14 resulted in severely collapsed xylem vessels with altered secondary wall structure, and an

15 extremely retarded plant growth. These findings indicate that TBL34 and TBL35 are putative

16 acetyltransferases required for xylan 3-O-monoacetylation and 2,3-di-O-acetylation and that

17 xylan acetylation is essential for normal secondary wall deposition and plant growth.

18 Keywords: Acetylation - Arabidopsis - DUF231 - secondary wall - xylan 


\section{Introduction}

Xylan is one of the major polysaccharides in lignocellulosic biomass targeted for biofuel production. It is made of a linear chain of $\beta-1,4-$ linked xylosyl residues, some of which are

4 substituted with glucuronic acid (GlcA), 4-O-methylglucuronic acid (MeGlcA), or arabinose

5 depending on plant species [1]. Xylosyl residues in xylan may also be acetylated at $O-2$ and/or

$6-3$ [2]. The reducing end of xylan in gymnosperms and dicots contains a unique tetrasaccharide

7 sequence, $\beta$-D-Xyl-( $1 \rightarrow 3)$ - $\alpha$-L-Rha- $(1 \rightarrow 2)-\alpha$-D-GalA-( $1 \rightarrow 4)-\mathrm{D}-\mathrm{Xyl}$ [3-7]. Although xylan is

8 essential for the normal biosynthesis and assembly of secondary walls, its presence contributes to

9 biomass recalcitrance for biofuel production [8]. Xylan not only hinders enzymatic hydrolysis of

10 lignocellulosic biomass for sugar release [9] but also releases acetate from the acetyl groups during biomass pretreatments, which is inhibitory to microorganisms used for fermentation of sugars [10]. Hence, it is important to have a thorough understanding of how xylan is synthesized, which may provide genetic tools for custom-designing lignocellulosic biomass tailored for biofuel production.

Xylan biosynthesis entails enzymatic activities of a number of glycosyltransferases, methyltransferases, and acetyltransferases that mediate xylan backbone elongation and

7 substitutions [11]. In Arabidopsis, the elongation of the xylan backbone involves four GT43

8 family members [IRREGULAR XYLEM9 (IRX9), IRX9 HOMOLOG (I9H)/ IRX9-LIKE

9 (IRX9-L), IRX14, and I14H/IRX14-L] and two GT47 family members (IRX10 and IRX10-L).

20 Mutations of these glycosyltransferases cause severe reductions in xylosyltransferase activity, xylan backbone chain length, and xylan content [6,12-17]. Recombinant IRX10 and IRX10-L expressed in yeast or human cells display xylosyltransferase activity that is able to transfer xylosyl residues onto xylooligomer acceptors $[18,19]$. Although recombinant GT43 proteins 
1 expressed in yeast do not exhibit xylosyltransferase activity, microsomes from tobacco BY2 cells

2 co-expressing IRX9 and IRX14 exhibit a high level of xylosyltransferase activity that is capable

3 of adding xylosyl residues onto xylooligomer acceptors [20]. GlcA substitution of xylan is

4 mediated by three GT8 proteins, GlucUronic acid substitution of Xylan1 (GUX1), GUX2, and

5 GUX3, as their simultaneous mutations result in a complete loss of GlcA side chains in xylan

6 [21,22]. Methylation of the GlcA side chains in xylan is carried out by three DUF579-containing

7 proteins, GLUCURONOXYLAN METHYLTRANSFERASE1 (GXM1), GXM2 and

8 GXM3/GXMT1, and their simultaneous mutations lead to a complete loss of GlcA methylation

9 in xylan [23-25]. Several other glycosyltransferases, including FRAGILE FIBER8 (FRA8),

10 FRA8 HOMOLOG (F8H), IRX8 and PARVUS, have been shown to be involved in the

11 biosynthesis of xylan reducing end tetrasaccharide sequence [6,12,26,27,28].

REDUCED WALL ACETLATIONs (RWAs), ESK1/TBL29, and ALTERED

13 XYLOGLUCAN9 (AXY9) have been implicated in xylan acetylation. RWAs are close

14 homologs of the fungal capsule synthesis gene1 (Cas1p) that is essential for the acetylation of

15 capsule polysaccharide glucuronoxylomannan [29]. The four Arabidopsis RWA genes,

$16 R W A 1 / 2 / 3 / 4$, are expressed in secondary wall-forming cells and their simultaneous mutations

17 cause a $40 \%$ reduction in xylan acetyl content [30]. RWAs are proposed to be putative

18 transporters of acetyl donors used for polysaccharide acetylation since they only have multiple

19 transmembrane domains and lack a putative acetyltransferase domain [31]. Mutation of the $A X Y 9$

20 gene results in a reduction in the acetylation of both xyloglucan and xylan and, therefore, AXY9

21 is hypothesized to be involved in supplying acetyl donor substrates for acetylation of multiple

22 polysaccharides [32]. The ESK1 gene, which encodes a protein in the plant-specific DUF231

23 family [33], is specifically expressed in xylem vessels and fibers and its mutation causes a 
1 specific reduction in xylan 2-O- and 3-O-monoacetylation and a decrease in xylan

2 acetyltransferase activity [34,35]. In vitro activity assay has confirmed that recombinant ESK1

3 exhibits acetyltransferase activity capable of catalyzing 2-O- and 3-O-monoacetylation of

4 xylooligomers [19]. Because the esk1 mutation causes only a partial reduction in xylan 2-O-and

5 3-O-monoacetylation and has no effects on 2,3-di-O-acetylation or 3-O-acetylation of xylosyl

6 residues substituted at $O-2$ with GlcA, it has been proposed that close homologs of ESK1 are

7 candidates to be acetyltransferases involved in xylan acetylation [34]. In this report, we

8 demonstrate the involvement of Arabidopsis TBL34 and TBL35, two close homologs of ESK1,

9 in mediating 3-O-monoacetylation and 2,3-di-O-acetylation of xylan.

\section{2. Materials and methods}

\section{2.1. Plant growth conditions}

13 Wild type and mutant plants were grown in a greenhouse with $16-\mathrm{hr}$ light/8-hr dark cycle.

14 The plants were treated with fertilizer every two weeks. Both wild type and mutant plants were

15 grown simultaneously for observation of their morphological phenotypes and their stems were

16 collected for cell wall analysis.

\subsection{Gene expression analysis}

The following tissues were used for RNA isolation with a Qiagen RNA isolation kit (Qiagen). Wild-type leaves and roots were from 6-week-old plants. Wild-type stems from 6week-old plants were divided into top, middle, and bottom parts, which represent the rapidly elongating segments, segments near cessation of elongation, and non-elongating segments, respectively. The top parts of stems contained only protoxylem as examined by toluidine blue 
1 staining of stem cross sections, the middle parts developed both protoxylem xylem and

2 metaxylem but lacked thick walls in interfascicular fibers, and the bottom parts had thick walls in

3 interfascicular fibers. Only main inflorescence stems with branches removed were collected for

4 RNA isolation. For the SECONDARY WALL NAC DOMAIN PROTEIN1 (SND1)

5 overexpressors, leaves from 4-week-old plants were used. For the snd1 nst1 mutant, stems from

6 6-week-old plants were used. First strand cDNAs were synthesized from total RNA and then

7 used as a template for real-time quantitative PCR analysis with the QuantiTect SYBR Green

8 PCR kit (Clontech). The PCR primers for TBL34 were 5'-cattacgcaactatcagagtatcg-3' and 5'-

9 tcaactagaatgatggtcaagaat-3', and those for $T B L 35$ were 5' -agagattgggaccaaaggtctcag-3' and 5'-

10 tcacaaaaaatggaaaagcaattgattcc-3'. PCR threshold cycles for genes of interest were selected in the

11 beginning of their exponential phases on PCR reaction curves. The expression level of each gene

12 was calculated by normalizing their PCR threshold cycle numbers with those of the UBQ10

13 (At4g05320), GAPDH (Atlg13440), and EFl $\alpha(\square \mathrm{t} \square \square \square \square \square \square \square \square$ reference genes and

14 quantitated according to the instructions from the PCR cycler manufacturer (BIO-RAD). The

15 PCR primers for $U B Q 10$ were 5'-ggccttgtataatccctgatgaataag-3' and 5'-

16 aaagagataacaggaacggaaacatagt-3', those for $G A P D H$ were 5'-ttggtgacaacaggtcaagca-3' and 5'-

17 aaacttgtcgctcaatgcaatc-3', and those for EFl $\alpha$ were 5'-tgagcacgctcttcttgctttca-3' and 5'-

18 ggtggtggcatccatcttgttaca-3'. The data were the average of three biological replicates.

\section{3. $\beta$-Glucuronidase (GUS) reporter gene analysis}

22 sequences, including the 3-kb 5' upstream sequence, the entire coding region, and the 2-kb 3'

23 downstream sequence were used. The whole gene sequence of $T B L 34$ was retrieved from the 
1 BAC clone under GenBank accession number AC004683 and genomic locus number

2 T19C21.19, and that of TBL35 was retrieved from the BAC clone under GenBank accession

3 number AL161946 and genomic locus number F7A7_140. The GUS reporter gene was inserted

4 in frame just before the stop codon of the TBL34 and TBL35 genes, which were cloned in the

5 binary vector pBI101 (Clontech). The constructs were transformed into wild-type Arabidopsis

6 plants by Agrobacterium-mediated transformation [36]. The first generation of transgenic plants

7 was used to examin GUS activity as described previously [28]. At least 10 independent

8 transgenic plants were examined and a consistent GUS staining pattern was observed.

10 2.4. Subcellular localization

11 For subcellular localization, the full-length $T B L 34$ and $T B L 35$ cDNAs were ligated in

12 frame at the amino terminus of yellow fluorescent protein (YFP) in a high-copy pBI221 plasmid

13 vector (Clontech) to create YFP-tagged TBL34 and TBL35 expression constructs. The

14 expression of TBL34-YFP and TBL35-YFP was driven by the cauliflower mosaic virus $35 \mathrm{~S}$

15 promoter. They were co-expressed with cyan fluorescent protein (CFP)-tagged Golgi marker

16 FRA8 in Arabidopsis leaf protoplasts [37]. The FRA8-CFP construct was generated by ligating

17 the full-length FRA8 cDNA in frame at the amino terminus of CFP in the pBI221 vector [28].

18 The fluorescence signals were visualized using a Leica TCs SP2 spectral confocal microscope

19 (Leica Microsystems). The TMHMM2.0 program (http://www.cbs.dtu.dk/service/TMHMM-2.0)

20 [38] and the Golgi Predictor program (http://ccb.imb.uq.edu.au/golgi/golgi_predictor.shtml) [39]

21 were used to predict putative subscellular localizations of TBL34 and TBL35.

\section{2.5. Histology}


Stems and root-hypocotyls of the wild type and mutants were fixed in $2 \%$ formaldehyde

2 and embedded in Low Viscosity (Spurr's) resin (Electron Microscopy Sciences) as described

3 [40]. One- $\mu$ m-thick sections were cut with a microtome and stained with $0.01 \%$ toluidine blue.

4 Stems and root-hypocotyls from at least 4 plants were sectioned and representative data were

5 shown. The bottom 2-cm segments of inflorescence stems of mature plants (8-week-old wild

6 type, $t b l 34, t b l 35$ and tbl34 tbl35; 10-week-old esk1, tbl34 esk1 and tbl35 esk1; and 12-week-old

$7 \quad t b l 34$ tbl35 eskl) were measured for their breaking force using a digital force/length tester

8 (Larson System) [41]. The breaking force was measured as the force needed to break apart a

9 stem segment.

10

11 2.6. Cell wall sugar composition analysis

12 Mature inflorescence stems from pools of at least 30 Arabidopsis plants for each mutant

13 line were ground in liquid nitrogen and isolated for cell wall residues [28]. Cell wall sugars (as

14 alditol acetates) were determined following the procedure as described [42]. The alditol acetates

15 of the hydrolyzed cell wall sugars were analyzed on a PerkinElmer Clarus 500 gas-liquid

16 chromatograph instrument equipped with a $30 \mathrm{~m}$ x $0.25 \mathrm{~mm}$ (i.d.) silica capillary column DB 225

17 (Alltech Assoc.). The sugar standards, including glucose, xylose, mannose, arabinose and

18 rhamnose, were purchased from Sigma-Aldrich.

19

20 2.7. Quantitative measurement of acetyl groups

21 Acetylated xylan from stem cell wall residues was extracted with dimethyl sulfoxide

22 (DMSO) following the procedure described [43]. The amount of acetyl groups in DMSO-

23 extracted xylan was determined by quantifying acetic acid released from xylan upon treatment 
1 with $\mathrm{NaOH}$ [44]. The amount of acetic acid was determined using an acetic acid assay kit

2 (Megazyme), and acetic acid from the kit was used as a standard for quantitation according to the 3 protocol from Megazyme.

4

$5 \quad 2.8 .{ }^{1} \mathrm{H}$-nuclear magnetic resonance (NMR) spectroscopy

6 DMSO-extracted xylans from various mutants were digested with $\beta$-endoxylanase M6

7 (Megazyme) and subsequently analyzed by NMR spectroscopy. NMR spectra were acquired at

$820{ }^{\circ} \mathrm{C}$ on a $600 \mathrm{MHz}$ spectrometer $\left(599.7 \mathrm{MHz},{ }^{1} \mathrm{H}\right)$ using a $3 \mathrm{~mm}$ cryogenic triple resonance

9 probe [45]. The samples were prepared with $100 \% \mathrm{D}_{2} \mathrm{O}$, and 128 transients were collected for

10 each sample. The ${ }^{1} \mathrm{H}$ NMR assignments of resonances were established by comparing them to

11 the NMR spectral data for xylan structure [2,46]. For wild type and each mutant sample, xylan

12 was extracted from a pool of inflorescence stems of at least 30 individual plants. For the $t b l 34$

13 tbl35 esk1 triple mutant, inflorescence stems from a pool of 150 plants were used. NMR spectra

14 from three replicates were obtained for each sample and they showed nearly identical resonance

15 signals with less than 5\% variation. The observed difference between the wild type and mutants

16 was statistically significant $(p<0.001)$ using the Student's $t$ test program.

17

18 2.9. Statistical analysis

19 The data of quantitative PCR analysis, breaking force and cell wall chemical

20 analysis were examined using the Student's $t$ test program

21 (http://www.graphpad.com/quickcalcs/ttest1.cfm).

22

23 2.10. Accession numbers 
The Arabidopsis Genome Initiative locus identifiers for the Arabidopsis genes

2 investigated in this study are TBL34 (At2g38320), TBL35 (At5g01620), and ESK1

3 (At3g55990).

4

\section{3. Results}

$6 \quad$ 3.1. Xylem-specific expression of TBL34

7 TBL34 and TBL35 are among the nine Arabidopsis DUF231-containing proteins that are

8 phylogenetically closely related to ESK1 [33] (Fig. 1A). For amino acid sequences, TBL34 and

9 TBL35 exhibit $36 \%$ and $39 \%$ identity and $62 \%$ and $66 \%$ similarity to ESK1, respectively. ESK1

10 is an acetyltransferase involved in xylan 2-O- and 3-O-monoacetylation $[19,34]$. To investigate

11 the functions of TBL34 and TBL35, we first studied their gene expression patterns. Quantitative

12 PCR analysis showed that although TBL35 did not exhibit organ-specific expression, TBL34 was

13 preferentially expressed in the middle and bottom parts of stems (Fig. 1B), in which secondary

14 walls were heavily deposited in xylem cells and interfascicular fibers. In addition, the expression

15 of $T B L 34$ was induced by overexpression of SND1, and the expression of both TBL34 and

16 TBL35 was downregulated in the stems of the snd1 nst 1 double mutant (Fig. 1C). SND1 was

17 previously discovered to be a fiber-specific NAC domain transcription factor and together with

18 its functionally-redundant paralog NST1, it controls the activation of secondary wall biosynthesis

19 genes, leading to the deposition of secondary wall components, including cellulose, xylan and

20 lignin [47]. Simultaneous mutations of SND1 and NST1 have previously been shown to cause a

21 loss of secondary walls in both xylary fibers and interfascicular fibers [48]. The finding that

$22 T B L 34$ expression is regulated by SND1 indicates the involvement of TBL34 in secondary wall

23 biosynthesis. 
2 reporter gene revealed that while no GUS activity was detected in any tissues of TBL35::GUS

3 transgenic plants probably due to its low expression level, TBL34::GUS plants displayed strong

4 GUS expression in a tissue-specific manner. Intense GUS staining was observed in developing

5 vessels in the rapidly elongating segments of stems (Fig. 1D), which had secondary wall

6 thickening only in protoxylem as examined by toludine blue staining of stem sections. In the

7 segments near cessation of elongation, in which thin secondary walls were seen in interfascicular

8 fibers, and the non-elongating segments, in which thick secondary wall thickening occured in

9 both metaxylem and interfascicular fibers as examined by toludine blue staining of stem sections,

10 GUS staining was seen in vessels and xylary fibers in the metaxylem (Fig. 1E and F). Heavy

11 GUS staining was also observed in developing secondary xylem in root-hypocotyls (Fig. 1G).

12 The observation that TBL34 expression is xylem-specific provides further evidence suggesting

13 its association with secondary wall biosynthesis.

14

18 To verify the subcellular localization of TBL34 and TBL35, we co-expressed yellow fluorescent

19 protein (YFP)-tagged TBL34 and TBL35 together with cyan fluorescent protein (CFP)-tagged

20 Golgi marker FRA8 [28] in Arabidopsis leaf protoplasts. The experiment showed that protoplasts

21 expressing TBL34-YFP and TBL35-YFP exhibited punctate fluorescent signals that were

22 superimposed with those of FRA8-CFP (Fig. 2C-J), indicating that TBL34 and TBL35 are 
1 targeted to the Golgi, where xylan biosynthesis occurs. In contrast, protoplasts expressing YFP

2 alone had fluorescent signals throughout the cytoplasm (Fig. 2B).

4 3.3. Mutations of TBL34 and TBL35 in the esk1 background exacerbated the defects in plant

5 growth and vessel morphology

6 The T-DNA insertion mutants of TBL34 and TBL35 (Fig. 3A) and the esk1 mutant were

7 crossed to generate various double and triple mutants for examination of mutant phenotypes.

8 Although the single and double mutants of TBL34 and TBL35 did not show any alterations in

9 plant growth (Fig. 3B,C and E) and stem mechanical strength (Fig. 3F), their mutations in the

10 eskl background led to exacerbated defects in plant growth compared with the eskl mutant (Fig.

$113 \mathrm{~B}-\mathrm{F})$. In particular, the $t b l 34$ tbl35 esk 1 triple mutant displayed severely retarded growth of

12 inflorescence stems, and only very tiny inflorescences developed after prolonged growth (12

13 weeks; inset in Fig. 3D). In addition, stem strength was decreased in tbl34 esk1 and tbl34 tbl35

14 eskl compared with eskl (Fig. 3F).

15 Examination of xylem vessel morphology in stems of the single and double mutants of

$16 T B L 34$ and TBL35 revealed no alterations compared with the wild type (data not shown). The

17 esk1 mutant had a mild deformation of vessels in both stems and root-hypocotyls (Fig. 4B and

$18 \mathrm{H})$. Examination of $t b l 34$ tbl35 esk1 plants showed that the vessels in stems and root-hypocotyls

19 were more severely deformed than those in eskl (Fig. 4), which was consistent with the

20 predominant expression of TBL34 in xylem cells. Some of vessels were so severely collapsed

21 that they only had a small cavity (Fig. 4C and I). The exacerbated growth defect in tbl34tbl35

22 eskl could be attributed to reduced water conducting capacity conferred by such drastically

23 collapsed vessels. Transmission electron microscopy further revealed that although three distinct 
1 wall layers, namely S1, S2 and S3, were evident in the secondary walls of wild-type vessels (Fig.

$25 \mathrm{~A}$ ), such layers were absent in the secondary walls of vessels of eskl (Fig. 5B) and tbl34 tbl35

3 eskl (Fig. 5C). We also noted that the secondary walls of vessels in these mutants appeared to be

4 stained unevenly and the inner surface of the secondary walls was wavy compared with the wild

5 type. The reduction in the wall thickness of interfascicular fibers in the stems of tbl34 tbl35 esk1

6 plants compared with the wild type appeared to be similar to that in eskl (Fig. 4D-F).

7 Furthermore, although the amount of xylose was not altered, that of glucose was further reduced

8 in the stem cell walls of tbl34 tbl35 eskl compared to those of eskl (Fig. 6A). This further

9 reduction in glucose was also observed in the stem cell walls of tbl34 eskl and tbl35 eskl (Fig.

10 6A). Because the majority of glucose in stem cell walls is from cellulose, this finding suggests a

11 defect in cellulose deposition in these mutants.

\section{3.4. Involvement of TBL34 and TBL35 in xylan 3-O-monoacetylation and 2,3-di-O-acetylation}

14 To determine whether TBL34 and TBL35 perform similar functions as ESK1 in xylan

15 acetylation, we measured the acetyl contents in DMSO-extracted xylans from the single, double,

16 and triple mutants. Although the amount of acetyl groups in $t b l 35$ xylan was not significantly

17 altered compared to that of the wild-type xylan, those in $t b l 34$ and $t b l 34 t b l 35$ xylans were mildly

18 reduced (Fig. 6B). More severe reductions in acetyl groups were observed in tbl34 esk1, tbl35

19 eskl and tbl34 tbl35 eskl xylans, which contained 44\%, 53\% and 43\%, respectively, of the wild-

20 type acetyl level (Fig. 6B).

21 We next investigated the patterns of acetyl substitutions in xylans from the double and

22 triple mutants of $T B L 34, T B L 35$, and ESK1 using NMR spectroscopy. Examination of ${ }^{1} \mathrm{H}$ NMR

23 spectra showed that DMSO-extracted xylans from the wild type and various mutants exhibited 
1 resonances typical for acetyl groups between 2.1 and $2.3 \mathrm{ppm}$ and the sugar groups between 3.1

2 and 5.4 ppm (Fig. 7) [44]. Integration analysis showed that the relative amount of acetyl groups

3 in these mutants was reduced by various degrees compared with that in the wild type (Table 1).

4 Further examination of the fingerprint region of the ${ }^{1} \mathrm{H}$ NMR spetra of acetylated xylans revealed

5 specific defects in acetyl substitutions in the mutant xylans (Fig. 8). The assignment of proton

6 resonances for xylan structural fragments was based on published data [2,46]. Resonances

7 corresponding to various acetyl substitutions on xylosyl residues, including 2-O- and 3-O-

8 monoacetylated xylosyl residues, 2,3-di-O-acetylated xylosyl residues, and 3-O-monoacetylated

9 xylosyl residues substituted at $O-2$ with GlcA/MeGlcA were readily observed in the wild-type

10 and mutant xylans, albeit with different abundances (Fig. 8). Integration analysis showed that the

11 signal abundance for 3-O-monoacetylated xylosyl residues and 2,3-di-O-acetylated xylosyl

12 residues in tbl34 tbl35 was reduced down to $67 \%$ and $65 \%$, respectively, of that in the wild type

13 (Table 2). In contrast, no reduction in the signal abundance for 2-O-monoacetylated xylosyl

14 residues and 3-O-monoacetylated xylosyl residues substituted at $O-2$ with GlcA/MeGlcA was

15 observed in tbl34 tbl35; instead, their signal abundance was relatively elevated. This finding

16 indicates that TBL34 and TBL35 play a role in 3-O-monoacetylation and 2,3-di-O-acetylation of

17 xylan. The eskl mutation also caused a reduction in the signal abundance for 3-O-

18 monoacetylated xylosyl residues [34]. Consistently, simultaneous mutations of TBL34, TBL35

19 and ESK1 resulted in an exacerbated decrease in the signal abundance for 3-O-monoacetylated

20 xylosyl residues compared with the eskl mutation alone (Table 2). However, a residual amount

21 of 3-O-monoacetylated xylosyl residues remained in the xylan of tbl34 tbl35 esk1, indicating that

22 additional acetyltransferase activities are involved. It was interesting to note that the elevation in

23 the signal abundance for 2-O-monoacetylated xylosyl residues observed in tbl34 tbl35 was 
1 diminished in tbl34 tbl35 eskl, consistent with a role of ESK1 in 2-O-monoacetylation of xylan.

2 Similar to the eskl mutant, the tbl34 eskl, tbl35 eskl and tbl34 tbl35 esk1 mutants all had

3 elevated abundance of GlcA/MeGlcA (Table 2), which was likely attributed to the reduction of

4 2-O-acetylation, leaving more $O-2$ positions available for GlcA substitutions [34].

\section{4. Discussion}

9 rhamnogalacturonan I and rhamnogalacturonan II), and lignin [31,49]. In lignocellulosic biomass

10 targeted for biofuel production, xylan is one of the major polymers that is heavily acetylated. The

11 degree of acetyl substitutions in xylan from various species, including Arabidopsis, aspen,

12 Paulownia elongate $\mathrm{x}$ fortune, birch, beech, Eucalyptus globulus, Agave sisalana, ranges from

130.35 to $0.61[2,34,43,44,50-53]$. Acetyl substitutions in xylan can occur at $O-2$ or $O-3$ of

14 different xylosyl residues, at both $O-2$ and $O-3$ of the same xylosyl residue, and at $O-3$ of xylosyl

15 residues substituted at $O-2$ with GlcA/MeGlcA [2]. Recent studies have shown that ESK1 is an

16 acetyltransferase specifically catalyzing $2-O$ - and 3-O-monoacetylation of xylan $[19,34,34]$,

17 suggesting that different acetyltransferase activities mediate the regiospecific acetyl substitutions

18 of xylan. Our finding in this study that TBL34 and TBL35, two close homologs of ESK1, are

19 putative acetyltransferases involved in 3-O-monoacetylation and 2,3-di-O-acetylation of xylan

20 expands our understanding of the biochemical mechanism underlying xylan $O$-acetylation.

21 The fact that the $t b l 34$ tbl35 eskl triple mutant has a more severe reduction in xylan 3-O-

22 monoacetylation than either tbl34 tbl35 or esk1 alone indicates that TBL34, TBL35, and ESK1

23 function redundantly in xylan 3-O-monoacetylation. Despite this functional redundancy, TBL34 
1 and TBL35 differ from ESK1 in several respects. In addition to the reduction in xylan 3-O-

2 monoacetylation, simultaneous mutations of $T B L 34$ and $T B L 35$ also led to a reduction in xylan

3 2,3-di-O-acetylation, whereas the eskl mutation also resulted in a reduction in xylan 2-O-

4 monoacetylation. Furthermore, TBL34 is specifically expressed in xylem cells, whereas ESK1 is

5 expressed in both xylem cells and interfascicular fibers. It is interesting to find that the 2-O-

6 monoacetylation of xylosyl residues is elevated in the tbl34 tbl35 mutant and this elevation is

7 diminished in tbl34 tbl35 eskl. One plausible explanation is that xylan 2,3-di-O-acetylation may

8 be mediated by ESK1 and TBL34/35 in two steps: first, ESK1 catalyzes 2-O-acetylation of

9 xylosyl residues; second, TBL34/35 catalyze 3-O-acetylation of the already 2-O-acetylated

10 xylosyl residues. Therefore, mutations of $T B L 34$ and $T B L 35$ cause a reduction in the amount of

11 2,3-di-O-acetylation and a concomitant elevation in the amount of 2-O-monoacetylation. Since

12 mutation of ESK1 results in defective 2-O-acetylation, this elevation diminishes in $t b l 34$ tbl35

13 esk1.

14 Although we were unable to detect TBL35 expression using TBL35-GUS reporter gene

15 analysis, quantitative PCR analysis showed its expression in all organs (Fig. 1B). Chemical

16 analysis revealed that the tbl35 esk 1 double mutant had lower amounts of cell wall glucose (Fig.

17 6A), overall xylan acetyl groups (Fig. 6B), and 3-acetylated xylosyl residues compared with esk1

18 (Table 2), suggesting a role of TBL35 in xylan acetylation. It should also be pointed out that

19 although the tbl34 tbl35 esk 1 triple mutant had a significant reduction in 3-O-acetylation of

20 xylan, there remains a residual amount of 3-O-acetylated xylosyl residues, which implies that

21 additional acetyltransferase activities are involved in xylan 3-O-acetylation. There are six other

22 ESK1 close homologs in addition to TBL34 and TBL35 (Fig. 1A), all of which could be

23 involved in xylan acetylation. This possibility is consistent with our recent finding that two of 
1 them, TBL3 and TBL31, are also involved in xylan 3-O-acetylation [51], which may explain

2 why a residual amount of 3-O-acetylated xylosyl residues remains in the $t b l 34$ tbl35 eskl triple

3 mutant. More importantly, TBL34 is specifically expressed in xylem cells, and therefore its

4 mutation does not affect 3-O-acetylation of xylan in interfascicular fibers.

11 normal interaction with cellulose and the subsequent assembly of cellulose-xylan polymers in

12 secondary walls. As a result, the mutant has a substantial reduction in cellulose content. The fact

13 that the tbl34 tbl35 esk1 triple mutant has a much greater reduction in the amounts of both

14 cellulose and acetyl groups than the eskl mutant alone indicates a correlation between the

15 severity of defect in xylan acetylation and the reduction in cellulose content. The severe

16 deformation of vessels in tbl34 tbl35 eskl could be attributable to both the reduced cellulose

17 amount and the defective interaction between cellulose and mutant xylan. It is interesting to note

18 that the secondary walls of tbl34 tbl35 esk1 vessels lack distinct S1, S2 and S3 wall layers but

19 exhibit uneven staining pattern and wavy inner surface compared with those of wild-type vessels.

20 Because the layered appearance of secondary walls is determined by the orientated deposition of

21 cellulose microfibrils [11], the abnormal secondary wall structure observed in the mutant vessels

22 is very likely due to an altered deposition of cellulose microfibrils, which further indicates the

23 importance of xylan acetylation in secondary wall assembly. 
The Arabidopsis DUF231 family is a large, plant-specific family with 46 members [33].

2 So far, only a few of family members have been functionally characterized; these include

3 PMR5/TBL44 whose mutation increases resistance to powdery mildew fungi [57], TBR involved

4 in cellulose biosynthesis [33], AXY4/TBL27 and AXY4L/TBL22 required for xyloglucan

5 acetylation [58], and ESK1/TBL29, TBL3 and TBL31 essential for xylan acetylation [34,35,51].

6 Our finding that TBL34 and TBL35 are involved in xylan acetylation expands our understanding

7 of the functions of DUF231 members and lends further support to the hypothesis that members

8 of the DUF231 family mediate acetylation of different wall polymers [58]. Deciphering the

9 mechanisms underlying the acetylation of cell wall polymers will provide potential tools for

10 genetic modification of biomass composition better suited for biofuel production.

11

\section{Acknowledgments}

13 We thank the Arabidopsis Biological Resource Center for the $t b l 34$ and tbl35 T-DNA

14 insertion mutant lines, Ms. Elizabeth Richardson for transmission electron microscopy and two

15 anonymous reviewers for their constructive comments for improvement of the original

16 manuscript. This work was funded by the U.S. Department of Energy, Office of Science, Office

17 of Basic Energy Sciences through Grant DE-FG02-03ER15415. 


\section{$1 \quad$ Figure legends}

2 Fig. 1. Expression analyses of the TBL34 and TBL35 genes. (A) Phylogenetic relationship of

3 TBL34, TBL35, and other ESK1 homologs in the ESK1 subgroup of the Arabidopsis DUF231

4 family. TBR is another DUF231 member involved in cellulose deposition in trichomes. The

5 phylogenetic tree was constructed with the neighbor-joining algorithm. The 0.1 scale denotes

$610 \%$ change, and the bootstrap values resulted from 1,000 replicates are presented in percentages

7 at the nodes. (B) and (C) Quantitative PCR analysis of the expression of TBL34 and TBL35.

8 Shown are their expression levels in different Arabidopsis organs (B), in the SND1

9 overexpressors (SND1-OE) (C, left panel), and in the snd1 nst 1 double mutant (C, right panel).

10 The expression of each gene in the organ was presented as transcript copy number per ng total

11 RNA (B). The change in the expression of TBL34 and TBL35 in SND1 overexpressors (C, left

12 panel) and the sndl nst 1 double mutant (C, right panel) was compared with the wild type by

13 setting the expression level in the wild type as 1 for SND1 overexpressors and as 100 for snd 1

$14 n s t 1$. Error bars denote SD of three biological replicates. Asterisks denote statistically significant

15 differences compared with the wild type ( $p<0.001)$. (D) to (G) Cell-type expression analysis of

$16 T B L 34$ in Arabidopsis inflorescence stems and root-hypocotyls. The TBL34 gene fused with the

17 GUS reporter genes was introduced into Arabidopsis and the transgenic plants were examined

18 for GUS activity (shown as blue). (D) Cross section of the top stem showing GUS staining in the

19 vessels in the protoxylem. (E) and (F) Cross sections of the middle stem (E) and the bottom stem

20 (F) showing GUS staining in the vessels and xylary fibers in the metaxylem. (G) Cross section of

21 the root-hypocotyl showing GUS staining in the developing secondary xylem. co, cortex; if,

22 interfascicular fiber; pi, pith; sp, secondary phloem; sx, secondary xylem; ve, vessel; xf, xylary

23 fiber; xy, xylem. Bars $=266 \mu \mathrm{m}$. 
2 Fig. 2. Subcellular localization of fluorescent protein-tagged TBL34 and TBL35 in Arabidopsis

3 protoplasts. TBL34 and TBL35 fused with YFP were co-expressed with the CFP-tagged Golgi

4 marker FRA8 in Arabidopsis leaf protoplasts and their fluorescent signals were examined with a

5 laser confocal microscope. (A) TBL34 and TBL35 are type II membrane proteins as predicted by

6 the TMHMM2.0 program. Inside, the cytoplasmic side of the membrane; outside, the Golgi

7 lumen side of the membrane. (B) Distribution of the fluorescent signals throughout the

8 cytoplasm in an Arabidopsis protoplast expressing YFP alone. (C) to (F) Superimposition of the

9 punctate fluorescent signals (F) in an Arabidopsis protoplast (C) co-expressing TBL34-YFP (D)

10 and the Golgi-localized FRA8-CFP (E). (G) to (J) Superimposition of the punctate fluorescent

11 signals $(\mathrm{J})$ in an Arabidopsis protoplast $(\mathrm{G})$ co-expressing TBL35-YFP (H) and FRA8-CFP (I).

12 Bars in $(B)$ to $(\mathrm{J})=8 \mu \mathrm{m}$.

13

14 Fig. 3. Effects of mutations of TBL34, TBL35 and ESK1 on plant growth and stem mechanical

15 strength. (A) Diagrams showing the sites of T-DNA insertions in the TBL34 and TBL35 genes.

16 Filled boxes represent exons. (B) and (C) Morphology of 4-week-old (B) and 8-week-old (C)

17 plants of the wild type and various mutants. (D) Morphology of 10-week-old mutant plants. Inset

18 shows the image of a 12-week-old plant of $t b l 34$ tbl35 esk1. (E) Measurement of inflorescence

19 stem heights of the wild type and various mutant plants at different time points. (F) Measurement

20 of the breaking force of stems of the wild type and various mutants. Error bars represent SD of 20

21 plants. Asterisks denote statistically significant differences compared with the wild type ( $p<$ $220.001)$. 
1 Fig. 4. Simultaneous mutations of $T B L 34, T B L 35$ and $E S K 1$ result in severely collapsed xylem

2 vessels. The bottom segments of stems and the root-hypocotyls of 8-week-old wild-type, 10-

3 week-old esk1, and 12-week-old tbl34 tbl35 esk1 plants were sectioned and stained with

4 toluidine blue for examination of anatomical structures. (A) to (C) Cross sections of xylem

5 bundles of stems showing deformed vessels (arrows) in eskl (B) and tbl34 tbl35 eskl (C)

6 compared with the wild type (A). (D) to (F) Cross sections of the interfascicular regions of stems

7 showing a reduction in secondary wall thickness in eskl (E) and tbl34 tbl35 eskl (F) compared

8 with the wild type (D). (G) to (I) Cross sections of root-hypocotyls showing deformed vessels

9 (arrows) in eskl (H) and tbl34 tbl35 eskl (I) compared with the wild type (G). if, interfascicular

10 fiber; sx, secondary xylem; xy, xylem. Bar in (A) $=72 \mu \mathrm{m}$ for (A) to (I).

12 Fig. 5. Transmission electron micrographs of vessel secondary walls of wild type (A), esk1 (B)

13 and tbl34 tbl35 eskl (C). Ultrathin sections from stems were stained with uranyl acetate and lead

14 citrate, and observed using a transmission electron microscope. The numbers, 1, 2 and 3, marked

15 in the secondary walls of wild-type vessels (A) indicate the three distinct layers, S1, S2 and S3,

16 respectively. Note the lack of distinct layers, abnormal staining pattern and wavy inner surface of

17 secondary walls of eskl (B) and tbl34 tbl35 eskl (C) vessels compared with the secondary walls

18 of wild-type vessels (A). ve, vessel; xf, xylary fiber. Scale bar $=3 \mu \mathrm{m}$.

20 Fig. 6. Cell wall sugar composition and xylan acetyl contents in the wild type and various

21 mutants. Cell wall residues and xylan were extracted from the inflorescence stems of 8-week-old

22 wild-type, 9-week-old esk1, and 12-week-old tbl34 tbl35 esk1 plants. (A) Measurement of

23 neutral sugar composition in the cell walls of the wild type and various mutants. (B) 
1 Measurement of acetyl contents in the xylans of the wild type and various mutants. Error bars

2 denote SD of three biological samples. Asterisks denote statistically significant differences

3 compared with the wild type $(p<0.001)$.

4

$5 \quad$ Fig. 7. ${ }^{1} \mathrm{H}$ NMR spectra of DMSO-extracted xylans from the wild type, tbl34 tbl35, eskl, tbl34

6 eskl, tbl35 eskl, and tbl34 tbl35 eskl. The resonance regions for carbohydrate and acetyl groups

7 are indicated. HDO, hydrogen deuterium oxide.

9 Fig. 8. Diagram of an acetylated xylooligomer from wild-type Arabidopsis xylan (A) and the

10 fingerprint regions of the ${ }^{1} \mathrm{H}$ NMR spectra of DMSO-extracted xylans from the wild type, $t b l 34$

11 tbl35, eskl, tbl34 eskl, tbl35 eskl, and tbl34 tbl35 eskl (B). The resonances for non-acetylated

12 xylosyl residues (Xyl), 2-O-acetylated xylosyl residues (Xyl-2Ac), 3-O-acetylated xylosyl

13 residues (Xyl-3Ac), 2,3-di-O-acetylated xylosyl residues (Xyl-2,3Ac), 3-O-acetylated xylosyl

14 residues substituted at $O-2$ with GlcA/MeGlcA (Xyl-3Ac-2GlcA), and GlcA/MeGlcA are

15 marked. $\mathrm{H} 1, \mathrm{H} 2$ and $\mathrm{H} 3$ indicate the protons attached to carbon at positions 1, 2 and 3,

16 respectively, of the xylose ring. 
8 Table 1. Relative integrated values of acetyl groups and carbohydrates in DMSO-extracted

9 xylans from the wild type and various mutants

10

13

14

15

$$
16
$$

17

18

19

19 tbl34 tbl35 esk1 1

0.12

0.12

0.38

$\mathrm{DS}_{\mathrm{AC}}$

Relative amount

of acetyl groups ${ }^{c}$

20

$21{ }^{\mathrm{a}}$ The integration of carbohydrates was calculated from the NMR resonance between 3 and 5.5

$22 \operatorname{ppm}$ (Fig. 7) and taken as 1. 
$1{ }^{\mathrm{b}}$ The integration of acetyl groups was determined from the ratio of the NMR resonance of acetyl

2 groups to that of carbohydrates.

$3{ }^{\mathrm{c}}$ The amount of acetyl groups in the wild-type xylan is taken as 100 , and the relative amount of

4 acetyl groups in the mutants is calculated from the ratio of acetyl groups in the mutants over that

5 of the wild type.

$6 \quad \mathrm{DS}_{\mathrm{AC}}$, degree of substitution of xylosyl residues by acetyl groups. The $\mathrm{DS}_{\mathrm{AC}}$ values were

7 calculated by dividing the molar values of acetyl groups by those of carbohydrates (represented

8 by xylose) according to Telemen et al. [44].

9 Table 2 Relative content of acetyl groups in xylans from the wild type and various mutants

19 (Xyl-2,3Ac)

20 3-O-Acetylated, 2-O-GlcA- 7

12

10

21 substituted xylose

22 (Xyl-3Ac-2GlcA)

23 GlcA/MeGlcA

8

9

$14 \quad 17$

17

17 
$1 \quad$ Non-acetylated and 2-O-monoacetylated xylosyl residues are integrated based on their $\mathrm{H} 1$

2 anomeric proton resonances, and 3-O-monoacetylated, 2,3-di-O-acetylated and 3-O-acetylated 2-

$3 \quad O$-GlcA/MeGlcA-substituted xylosyl residues are integrated based on their $\mathrm{H} 3$ resonances (Fig.

4 8). The relative abundance (mol\%) of each structural fragment was calculated by first dividing

5 the integration value by its molecular mass and then by the molar values of total structural

6 fragments according to Teleman et al. [44]. The total balance of acetyl groups was verified by

$7 \quad$ integration of acetyl signals at $2.2 \mathrm{ppm}$ (Fig. 7).

8

9

\section{References}

11 [1] T.E. Timell, Recent progress in the chemistry of wood hemicelluloses, Wood Sci. Technol. 1

12 (1967) 45-70.

13 [2] A. Teleman, J. Lundqvist, F. Tjerneld, H. Stålbrand, O. Dahlman, Characterization of

14 acetylated 4- $O$-methylglucuronoxylan isolated from aspen employing ${ }^{1} \mathrm{H}$ and ${ }^{13} \mathrm{C} \mathrm{NMR}$

15 spectroscopy, Carbohydr. Res. 329 (2000) 807-815.

16 [3] K. Shimizu, M. Ishihara, T. Ishihara, Hemicellulases of brown rotting fungus,

17 Tyromyces palustris. II. The oligosaccharides from the hydrolysate of a hardwood xylan

18 by the intracellular xylanase, Mokuzai Gaikkashi 22 (1976) 618-625.

19 [4] M.H. Johansson, O. Samuelson, Reducing end groups in birch xylan and their alkaline

20 degradation, Wood Sci. Technol. 11 (1977) 251-263.

21 [5] S.-I. Andersson, O. Samuelson, M. Ishihara, K. Shimizu, Structure of the reducing

22 end-groups in spruce xylan, Carbohydr. Res. 111 (1983) 283-288. 
1 [6] M.J. Pena, R. Zhong, G.-K. Zhou, E.A. Richardson, M.A. O’Neill, A.G. Darvill, et al.,

2 Arabidopsis irregular xylem 8 and irregular xylem 9: implications for the complexity of

3 glucuronoxylan biosynthesis, Plant Cell 19 (2007) 549-563.

4 [7] C. Lee, Q. Teng, W. Huang, R. Zhong, Z.-H. Ye, Down-regulation of PoGT47C expression

5 in poplar results in a reduced glucuronoxylan content and an increased wood digestibility by

6 cellulase, Plant Cell Physiol. 50 (2009) 1075-1089.

7 [8] A. Carroll, C. Somerville, Cellulosic biofuels, Annu. Rev. Plant Biol. 60 (2009) 165-182.

8 [9] M.J. Selig, W.S. Adney, M.E. Himmel, S.R. Decker, The impact of cell wall acetylation on

9 corn stover hydrolysis by cellulolytic and xylanolytic enzymes, Cellulose 16 (2009) 711-722.

10 [10] S. Helle, D. Cameron, J. Lam, B. White, S. Duff, Effect of inhibitory compounds found in

11 biomass hydrolysates on growth and xylose fermentation by a genetically engineered strain of $S$.

12 cerevisiae, Enzyme Microb. Technol. 33 (2003) 786-792.

13 [11] R. Zhong, Z.-H. Ye, Secondary cell walls: biosynthesis, patterned deposition and

14 transcriptional regulation, Plant Cell Physiol. 56 (2015) 195-214.

15 [12] D.M. Brown, F. Goubet, V.W. Wong, R. Goodacre, E. Stephens, P. Dupree, et al.,

16 Comparison of five xylan synthesis mutants reveals new insight into the mechanisms of xylan

17 synthesis, Plant J. 52 (2007) 1154-1168.

18 [13] D.M. Brown, Z. Zhang, E. Stephens, P. Dupree, S.R. Turner, Characterization of IRX10 and

19 IRX10-like reveals an essential role in glucuronoxylan biosynthesis in Arabidopsis, Plant J. 57

$20 \quad$ (2009) 732-746.

21 [14] A.M. Wu, E. Hörnblad, A. Voxeur, L. Gerber, C. Rihouey, P. Lerouge, et al., Analysis of

22 the Arabidopsis IRX9/IRX9-L and IRX14/IRX14-L pairs of glycosyltransferase genes reveals 
1 critical contributions to biosynthesis of the hemicellulose glucuronoxylan, Plant Physiol. 153

2 (2010) 542-554.

3 [15] A.M. Wu, C. Rihouey, M. Seveno, E. Hörnblad, S.K. Singh, T. Matsunaga, et al., The

4 Arabidopsis IRX10 and IRX10-LIKE glycosyltransferases are critical for glucuronoxylan

5 biosynthesis during secondary cell wall formation, Plant J. 57 (2009) 718-731.

6 [16] C. Lee, M.A. O’Neill, Y. Tsumuraya, A.G. Darvill, Z.-H. Ye, The irregular xylem9 mutant

7 is deficient in xylan xylosyltransferase activity, Plant Cell Physiol. 48 (2007) 1624-1634.

8 [17] C. Lee, Q. Teng, W. Huang, R. Zhong, Z.-H. Ye, The Arabidopsis family GT43

9 glycosyltransferases form two functionally nonredundant groups essential for the elongation of

10 glucuronoxylan backbone, Plant Physiol. 153 (2010) 526-541.

11 [18] J.K. Jensen, N.R. Johnson, C.G. Wilkerson, Arabidopsis thaliana IRX10 and two related

12 proteins from Psyllium and Physcomitrella patens are xylan xylosyltransferases, Plant J. 80

13 (2014) 207-215.

14 [19] B.R. Urbanowicz, M.J. Peña, H.A. Moniz, K.W. Moremen, W.S. York, Two Arabidopsis

15 proteins synthesize acetylated xylan in vitro, Plant J. 80 (2014) 197-206.

16 [20] C. Lee, R. Zhong, Z.-H. Ye, Arabidopsis family GT43 members are xylan

17 xylosyltransferases required for the elongation of the xylan backbone. Plant Cell Physiol. 53

18 (2012) 135-143.

19 [21] J.C. Mortimer, G.P. Miles, D.M. Brown, Z. Zhang, M.P. Segura, T. Weimar, et al., Absence

20 of branches from xylan in Arabidopsis gux mutants reveals potential for simplification of

21 lignocellulosic biomass, Proc. Natl. Acad. Sci. USA 107 (2010) 17409-17414. 
1 [22] C. Lee, Q. Teng, R. Zhong, Z.-H. Ye, Arabidopsis GUX proteins are glucuronyltransferases

2 responsible for the addition of glucuronic acid side chains onto xylan, Plant Cell Physiol. 53

3 (2012) 1204-1216.

4 [23] C. Lee, Q. Teng, R. Zhong, Y. Yuan, M. Haghighat, Z.-H. Ye, Three Arabidopsis DUF579

5 domain-containing GXM proteins are methyltransferases catalyzing 4-O-methylation of

6 glucuronic acid on xylan, Plant Cell Physiol. 53 (2012) 1934-1949.

7 [24] B.R. Urbanowicz, M.J. Peña, S. Ratnaparkhe, U. Avci, J. Backe, H.F. Steet, et al., 4-O-

8 methylation of glucuronic acid in Arabidopsis glucuronoxylan is catalyzed by a domain of

9 unknown function family 579 protein, Proc. Natl. Acad. Sci. USA 109 (2012) 14253-14258.

10 [25] Y. Yuan, Q. Teng, C. Lee, R. Zhong, Z.-H. Ye, Modification of the degree of 4-O-

11 methylation of secondary wall glucuronoxylan, Plant Sci. 219-220 (2014) 42-50.

12 [26] C. Lee, R. Zhong, E.A. Richardson, D.S. Himmelsbach, B.T. McPhail, Z.-H. Ye, The

13 PARVUS gene is expressed in cells undergoing secondary wall thickening and is essential for

14 glucuronoxylan biosynthesis, Plant Cell Physiol. 48 (2007) 1659-1672.

15 [27] C. Lee, Q. Teng, W. Huang, R. Zhong, Z.-H. Ye, The F8H glycosyltransferase is a

16 functional paralog of FRA8 involved in glucuronoxylan biosynthesis in Arabidopsis, Plant Cell

17 Physiol. 50 (2009) 812-827.

18 [28] R. Zhong, M.J. Peña, G.-K. Zhou, C.J. Nairn, A. Wood-Jones, E.A. Richardson, et al.,

19 Arabidopsis Fragile Fiber8, which encodes a putative glucuronyltransferase, is essential for

20 normal secondary wall synthesis, Plant Cell 17 (2005) 3390-3408.

21 [29] G. Janbon, U. Himmelreich, F. Moyrand, L. Improvisi, F. Dromer, Cas 1p is a membrane

22 protein necessary for the $O$-acetylation of the Cryptococcus neoformans capsular polysaccharide,

23 Mol. Microbiol. 42 (2001) 453-467. 
2 ACETYLATION genes are expressed in secondary wall-containing cells and required for the

3 acetylation of xylan, Plant Cell Physiol. 52 (2011) 1289-1301.

4 [31] S. Gille, M. Pauly, $O$-acetylation of plant cell wall polysaccharides, Front. Plant Sci. 3

$5 \quad(2012) 12$.

6 [32] A. Schultink, D. Naylor, M. Dama, M. Pauly, The role of the plant-specific ALTERED

7 XYLOGLUCAN9 protein in Arabidopsis cell wall polysaccharide $O$-acetylation, Plant Physiol.

$8167(2015) 1271-1283$.

9 [33] V. Bischoff, S. Nita, L. Neumetzler, D. Schindelasch, A. Urbain, R. Eshed, et al.,

10 TRICHOME BIREFRINGENCE and its homolog AT5G01360 encode plant-specific DUF231

11 proteins required for cellulose biosynthesis in Arabidopsis, Plant Physiol. 153 (2010) 590-602.

12 [34] Y. Yuan, Q. Teng, R. Zhong, Z.-H. Ye, The Arabidopsis DUF231 domain-containing

13 protein ESK1 mediates 2-O-and 3-O-acetylation of xylosyl residues in xylan, Plant Cell Physiol.

$1454(2013) 1186-1199$.

15 [35] G. Xiong, K. Cheng, M. Pauly, Xylan $O$-acetylation impacts xylem development and

16 enzymatic recalcitrance as indicated by the Arabidopsis mutant tbl29, Mol. Plant 6 (2013) 1373-

$17 \quad 1375$.

18 [36] N. Bechtold, D. Bouchez, In planta Agrobacterium-mediated transformation of adult

19 Arabidopsis thaliana plants by vacuum infiltration. In Gene Transfer to Plants, I. Potrykus, G.

20 Spangenberg, eds (Berlin:Springer-Verlag), 1994, pp. 19-23.

21 [37] J. Sheen, Signal transduction in maize and Arabidopsis mesophyll protoplasts, Plant

22 Physiol. 127 (2001) 1466-1475. 
2 transmembrane domains, Bioinformatics 18 (2002) 1109-1115.

3 [39] A. Krogh, B. Larsson, G. von Heijne, E.L. Sonnhammer, Predicting transmembrane protein

4 topology with a hidden Markov model: application to complete genomes, J. Mol. Biol. 305

5 (2001) 567-580.

6 [40] D.H. Burk, R. Zhong, W.H.III Morrison, Z.-H. Ye, Disruption of cortical microtubules by

7 overexpression of green fluorescent protein-tagged $\alpha$-tubulin 6 causes a marked reduction in cell

8 wall synthesis, J. Integr. Plant Biol. 48 (2006) 85-98.

9 [41] R. Zhong, J.J. Taylor, Z.-H. Ye, Disruption of interfascicular fiber differentiation in an

10 Arabidopsis mutant, Plant Cell 9 (1997) 2159-2170.

11 [42] C. Hoebler, J.L. Barry, A. David, J. Delort-Laval, Rapid acid-hydrolysis of plant cell wall

12 polysaccharides and simplified quantitative determination of their neutral monosaccharides by

13 gas-liquid chromatography, J. Agr. Food Chem. 37 (1989) 360-367.

14 [43] V.M. Gonçalves, D.V. Evtuguin, M.R. Domingues, Structural characterization of the

15 acetylated heteroxylan from the natural hybrid Paulownia elongata/Paulownia fortune,

16 Carbohydr. Res. 343 (2008) 256-266.

17 [44] A. Teleman, M. Tenkanen, A. Jacobs, O. Dahlman, Characterization of O-acetyl-(4-O-

18 methylglucurono)xylan isolated from birch and beech, Carbohydr. Res. 337 (2002) 373-377.

19 [45] Q. Teng, D.R.Ekman, W. Huang, T.W. Collette, Push-through direct injection NMR: an

20 optimized automation method applied to metabolomics, Analyst 137 (2012) 2226-2232.

21 [46] R. Zhong, Q. Teng, C. Lee, Z.-H. Ye, Identification of a disaccharide side chain 2-O- $\alpha-D-$

22 galactopyranosyl- $\alpha-D$-glucuronic acid in Arabidopsis xylan, Plant Signal. Behav. 9 (2014)

23 e27933. 
2 regulator of secondary wall synthesis in fibers of Arabidopsis, Plant Cell 18 (2006) 3158-3170.

3 [48] R. Zhong, E.A. Richardson, Z.-H. Ye, Two NAC domain transcription factors, SND1 and

4 NST1, function redundantly in regulation of secondary wall synthesis in fibers of Arabidopsis,

$5 \quad$ Planta 225 (2007) 1603-1611.

6 [49] P.M.-A. Pawar, S. Koutaniemi, M. Tenkanen, E.J. Mellerowicz, Acetylation of woody

7 lignocellulose: significance and regulation, Front. Plant Sci. 4 (2013) 118.

8 [50] D.V. Evtuguin, J.L. Tomás, A.M. Silva, C.P. Neto, Characterization of an acetylated

9 heteroxylan from Eucalyptus globulus Labill, Carbohydr. Res. 338 (2003) 597-604.

10 [51] Y. Yuan, Q. Teng, R. Zhong, Z.-H. Ye, TBL3 and TBL31, two Arabidopsis DUF231

11 domain proteins, are required for 3-O-monoacetylation of xylan, Plant Cell Physiol. (in press).

12 [52] R. Naran, S. Black, S.R. Decker, P. Azadi, Extraction and characterization of native

13 heteroxylans from delignified corn stover and aspen, Cellulose 16 (2009) 661-675.

14 [53] G. Marques, A. Gutierrez, J.C. del Rio, D.V. Evtuguin, Acetylated heteroxylan from Agave

15 sisalana and its behavior in alkaline pulping and TCF/ECF bleaching, Carbohydr. Polymers 81

16 (2010) 517-523.

17 [54] S.O. Prozil, E.V. Costa, D.V. Evtuguin, L.P. Lopes, M.R. Domingues, Structural

18 characterization of polysaccharides isolated from grape stalks of Vitis vinifera L, Carbohydr.

19 Res. 356 (2012) 252-259.

20 [55] M. Gröndahl, A. Teleman, P. Gatenholm, Effect of acetylation on the material properties of

21 glucuronoxylan from aspen wood, Carbohydr. Polym. 52 (2003) 359-366.

22 [56] M.A. Kabel, H. van den Borne, J.-P. Vincken, A.G.J. Voragen, H.A. Schols, Structural

23 differences of xylans affect their interaction with cellulose, Carbohydr. Polym. 69 (2007) 94-105. 
1 [57] J.P. Vogel, T.K. Raab, C.R. Somerville, S.C. Somerville, Mutations in PMR5 result in

2 powdery mildew resistance and altered cell wall composition, Plant J. 40 (2004) 968-978

3 [58] S. Gille, A. de Souza, G. Xiong, M. Benz, K. Cheng, A. Schultink, I.B. Reca, M. Pauly, $O$ -

4 acetylation of Arabidopsis hemicellulose xyloglucan requires AXY4 or AXY4L, proteins with a 5 TBL and DUF231 domain, Plant Cell 23 (2011) 4041-4053.

6 

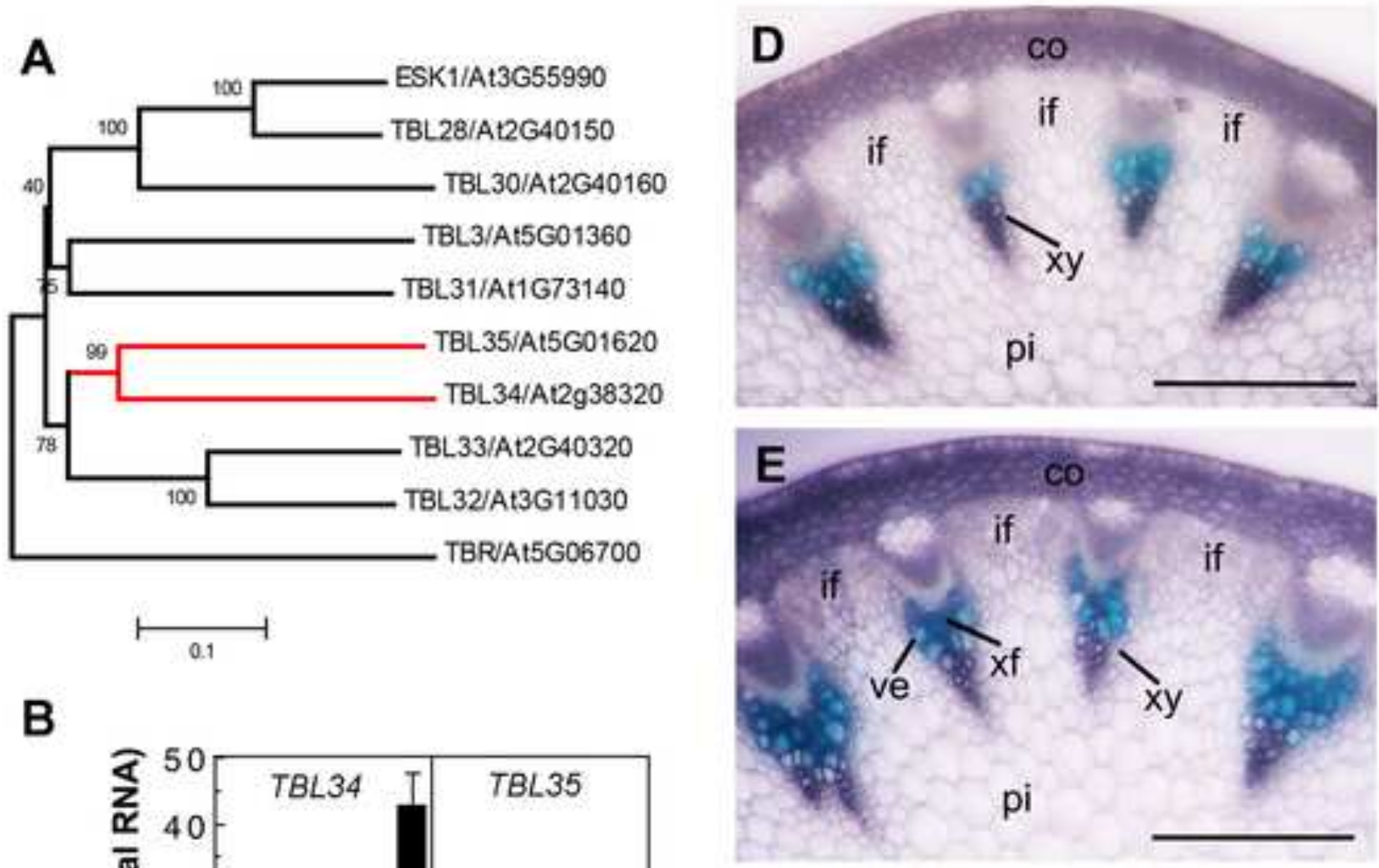

B

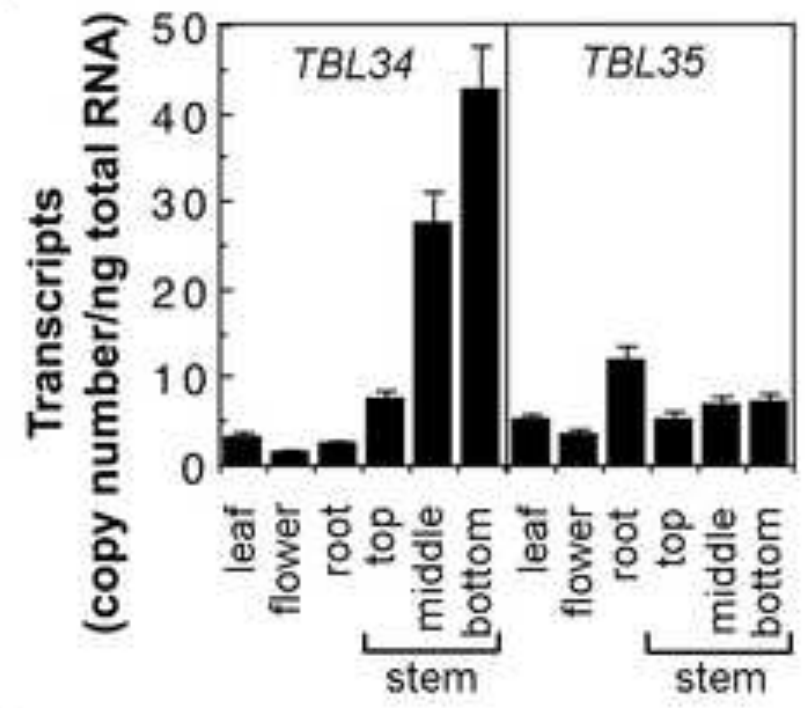

C
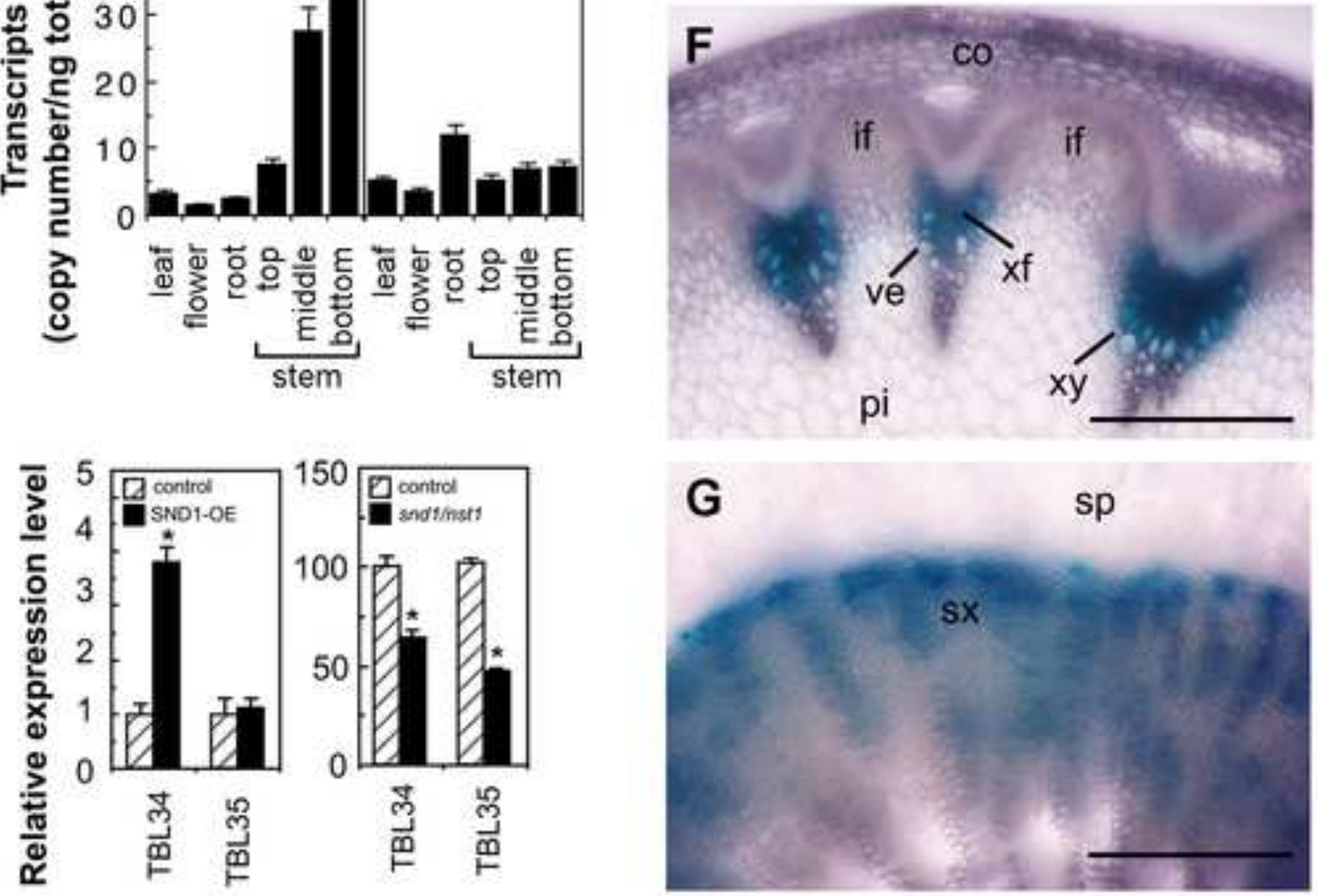
Transmumbrane - Inside - Outside -
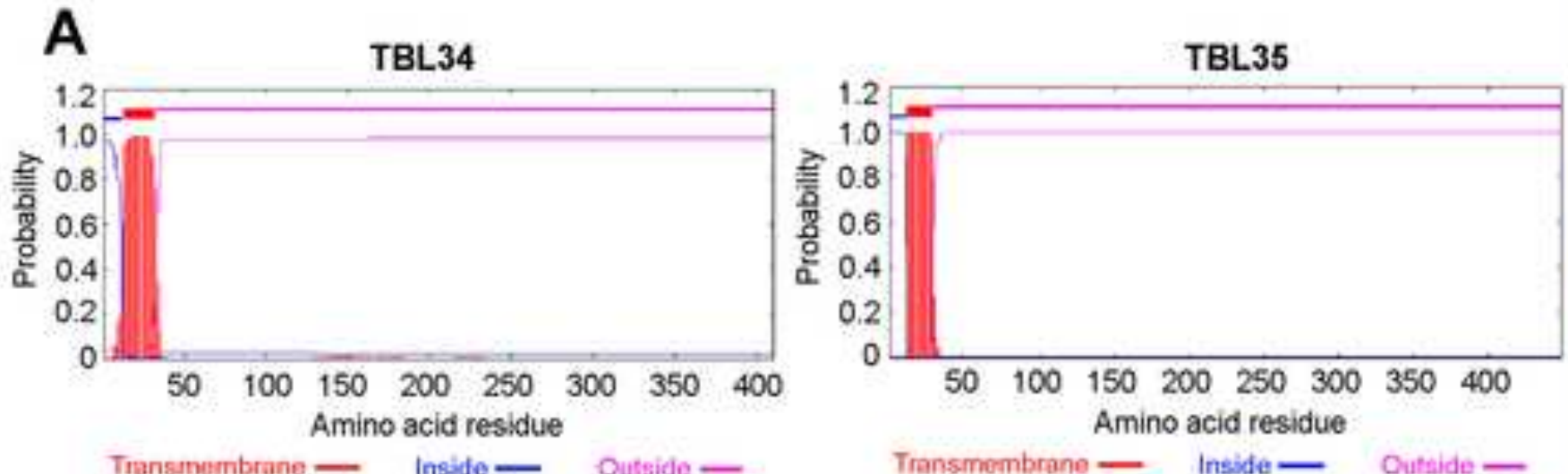

TBL34-YFP

D

,

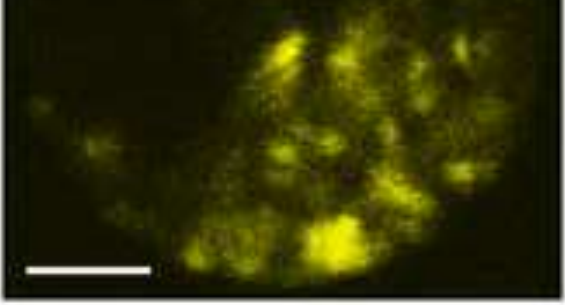

G

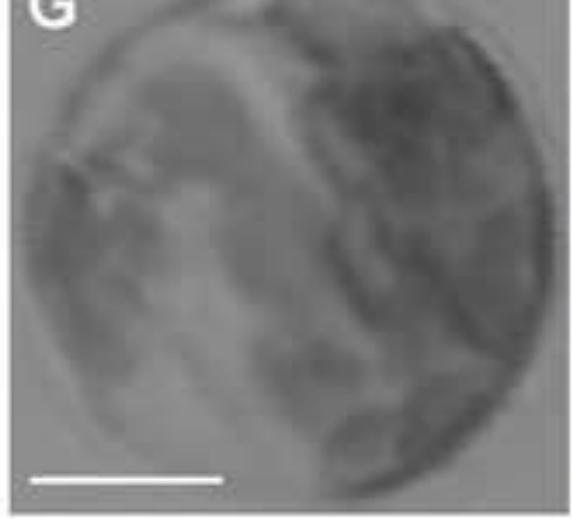

H

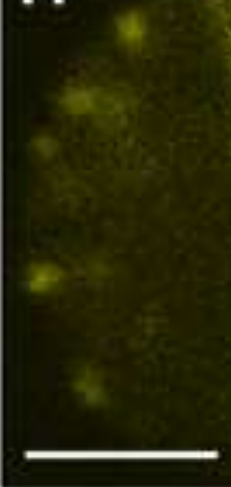

E

.
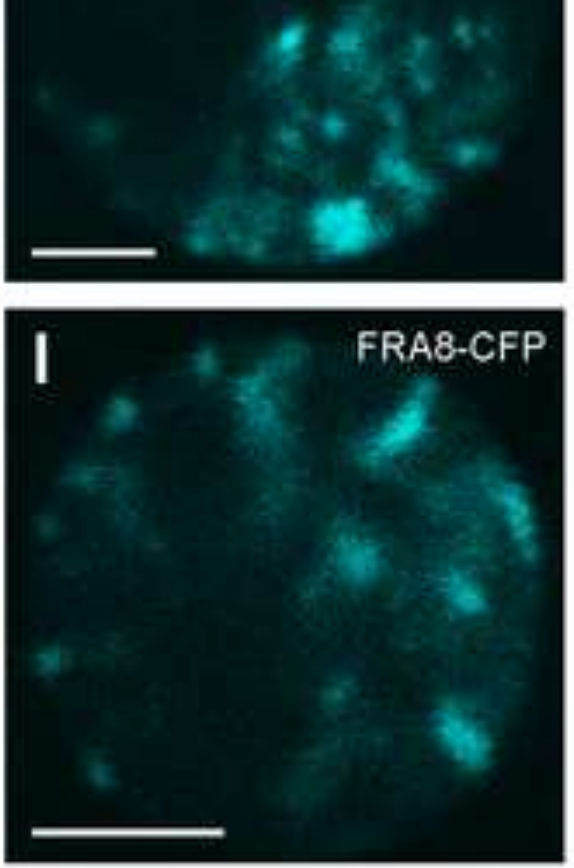

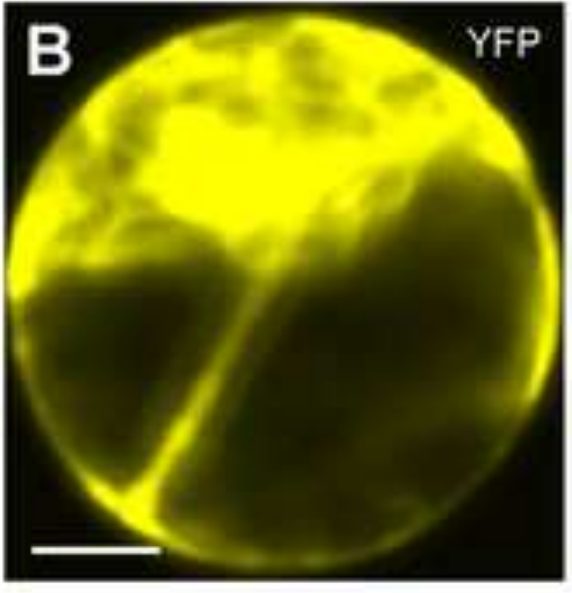

$F$

Merged

,
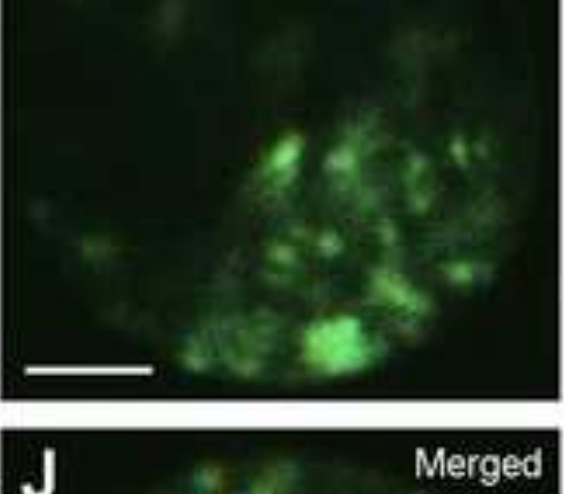

J

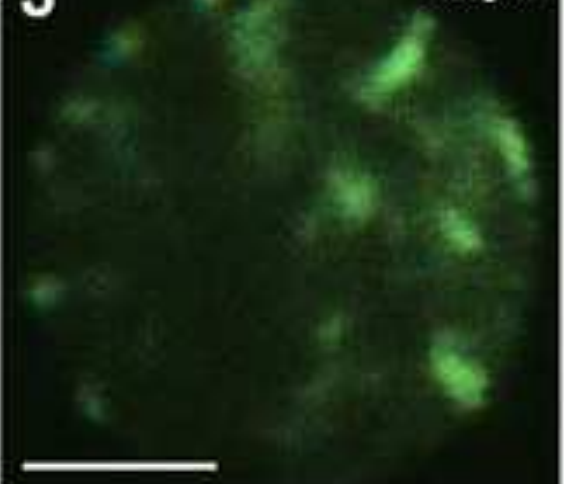


A
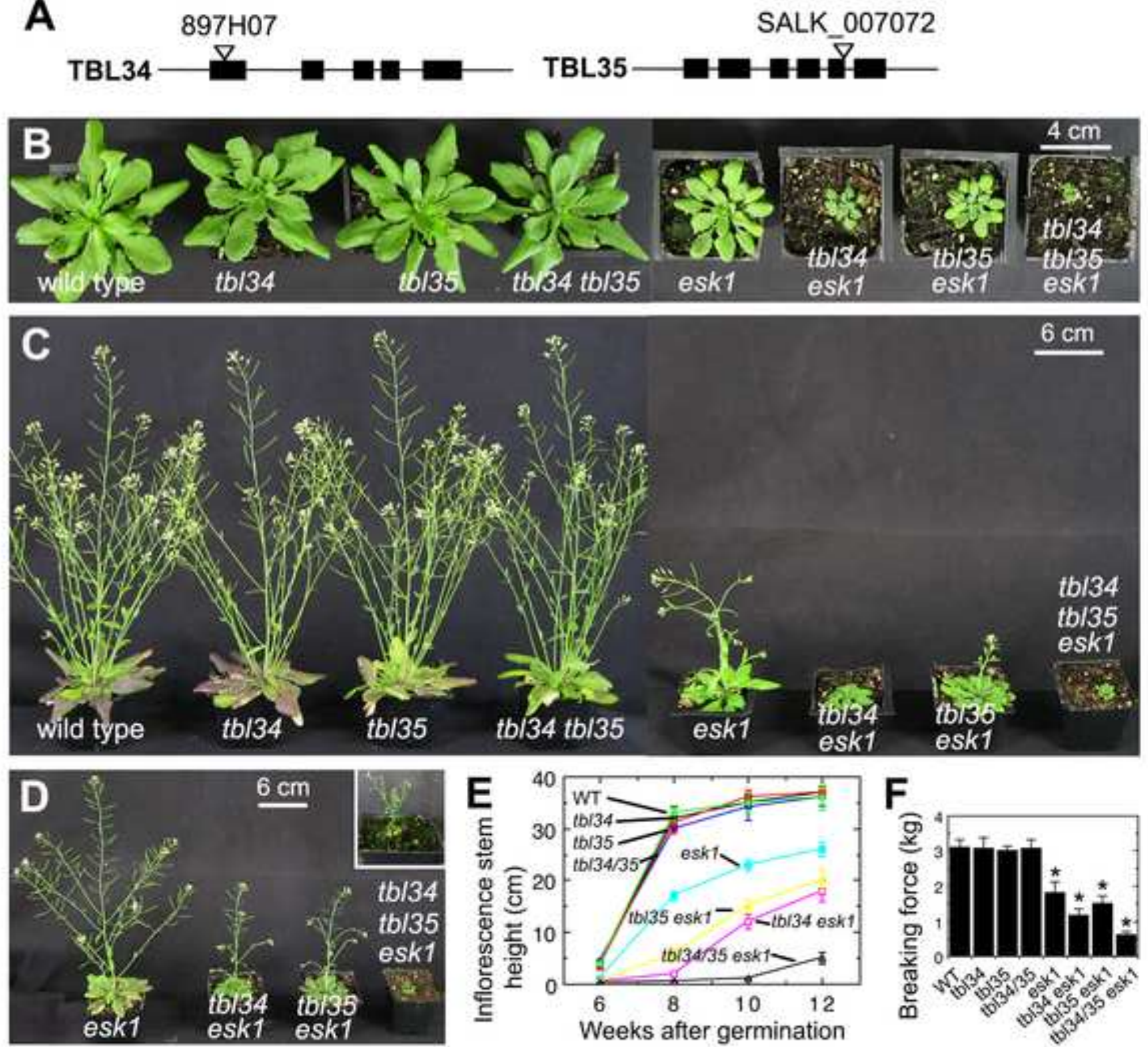
Wild type

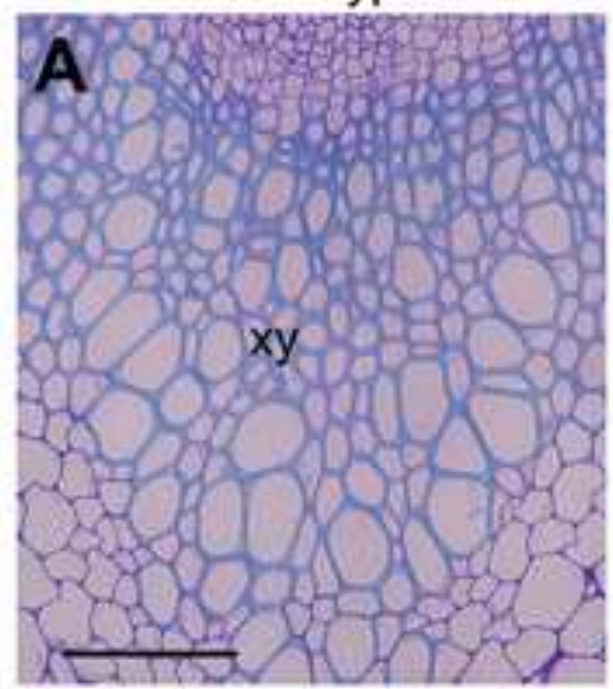

D
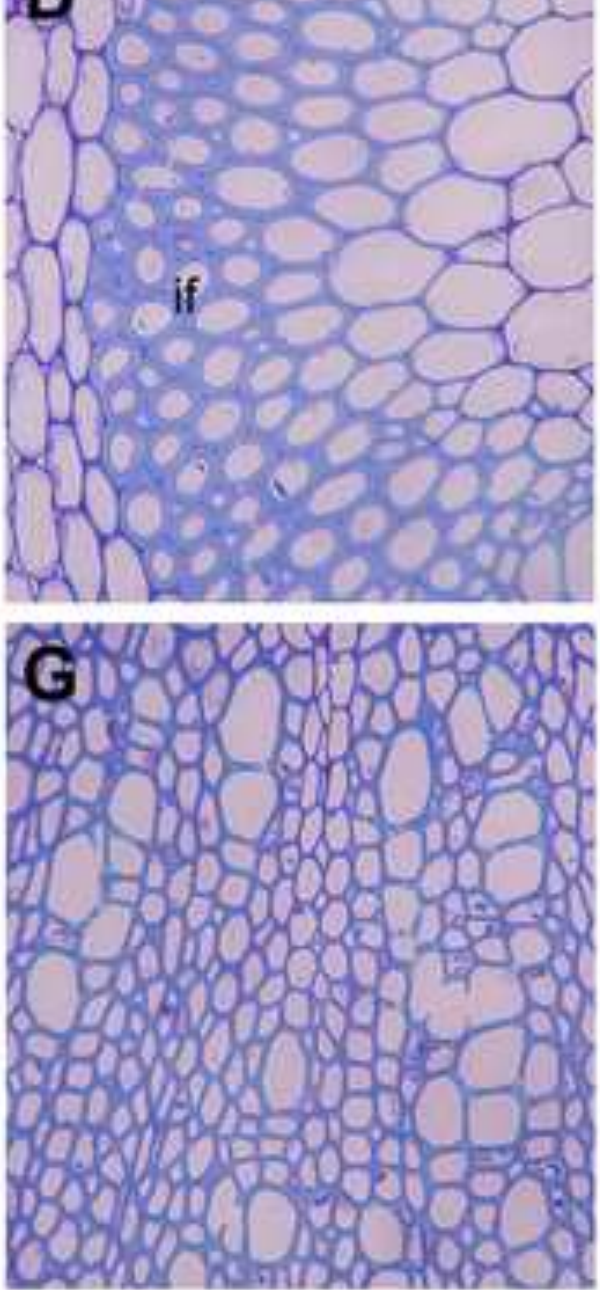

esk1
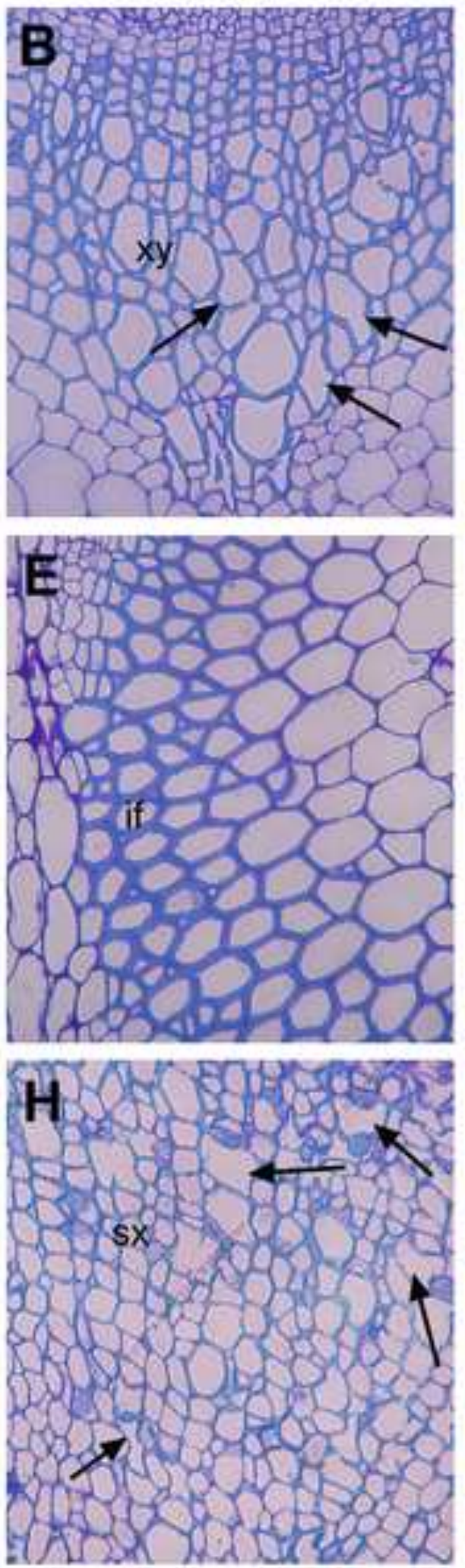

tb/34 tb/35 esk1
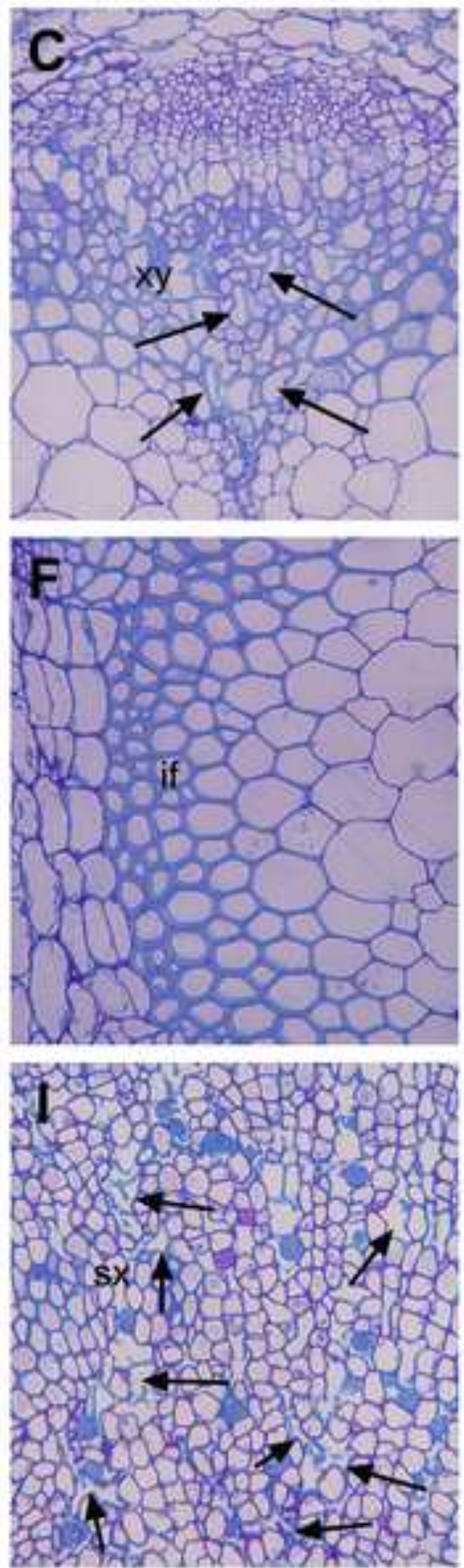


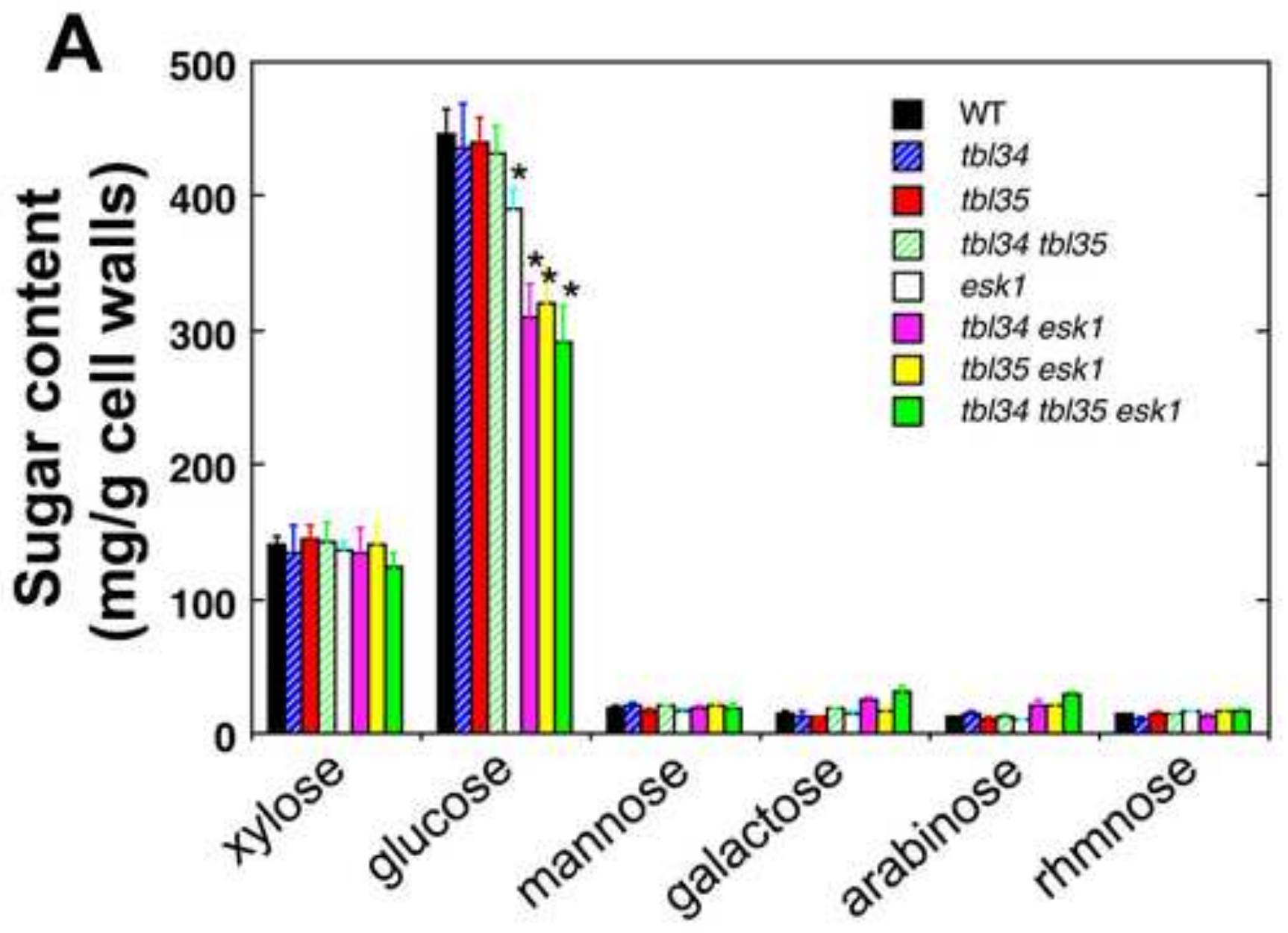

\section{B}

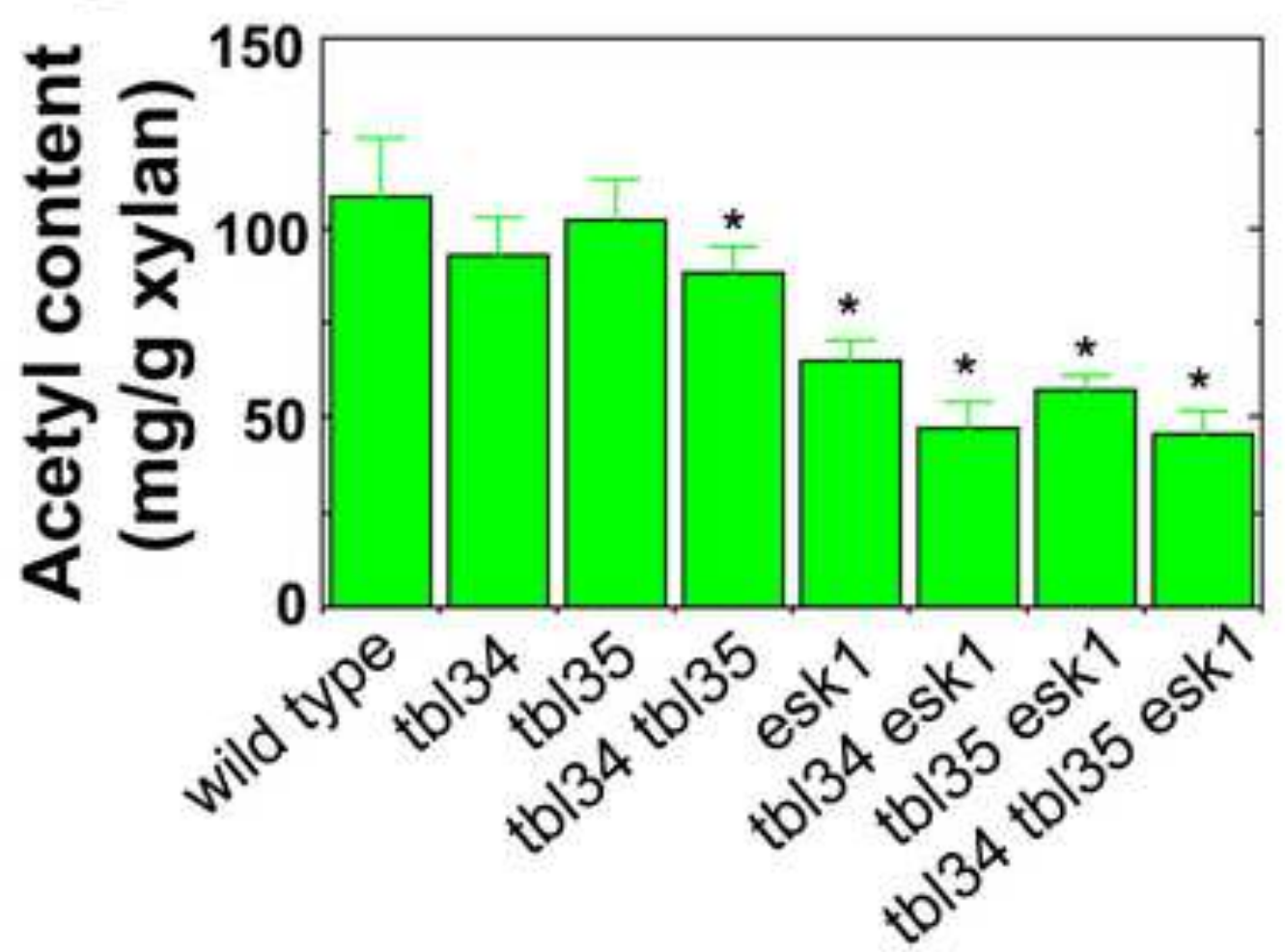




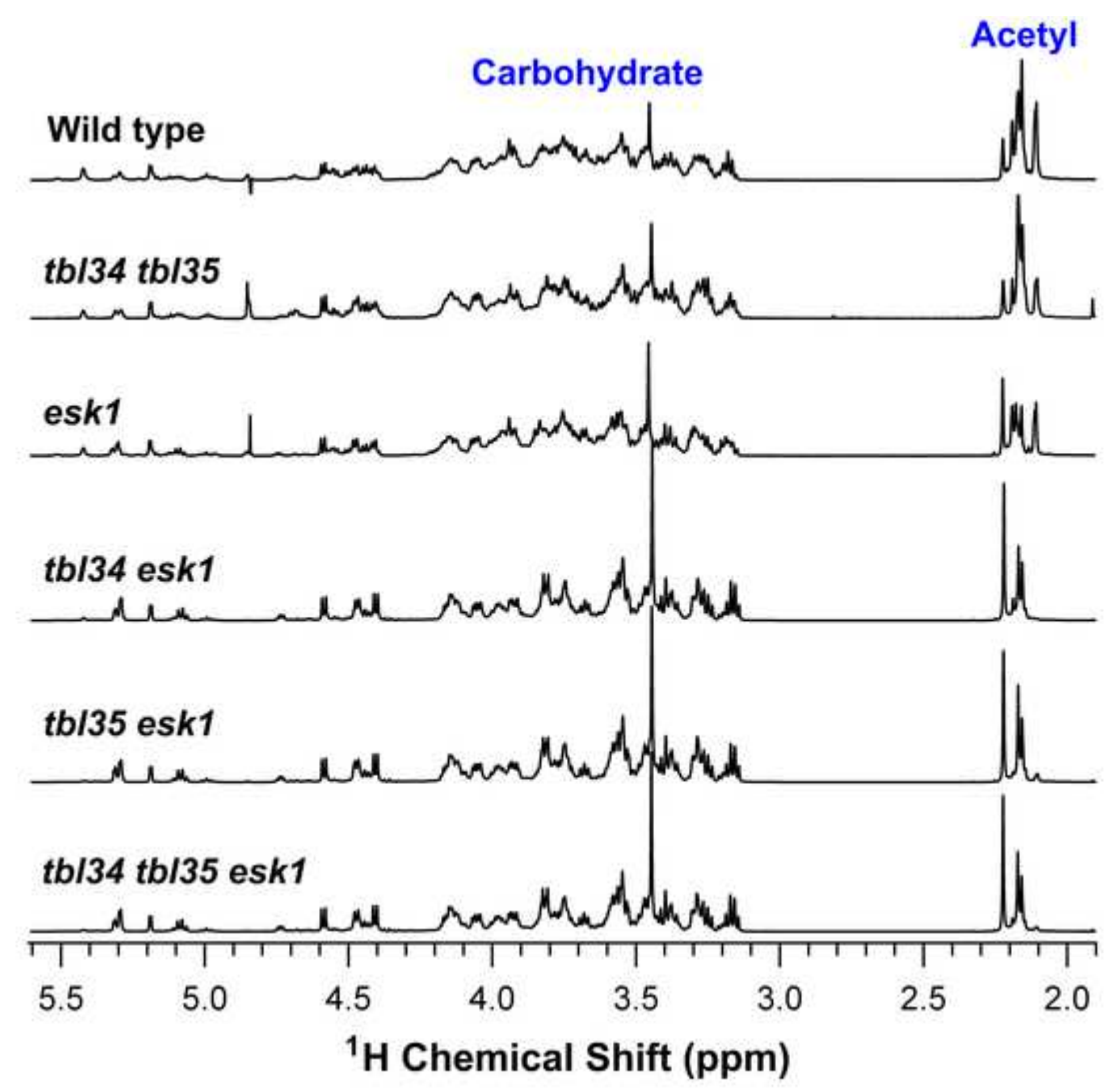


A

$\rightarrow 4)-\beta-D-X y l p-(1->4)-\beta-D-X y|p-(1->4)-\beta-D-X y l p-(1->4)-\beta-D-X y| p-(1->4)-\beta-D-X y l p-(1->$
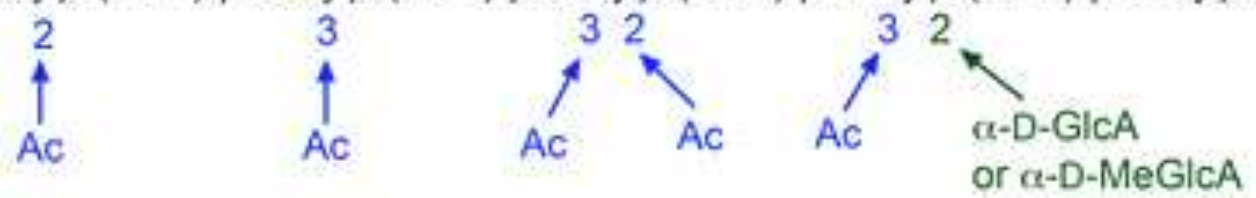

B

$\mathrm{H} 1$

$\mathrm{H} 1$

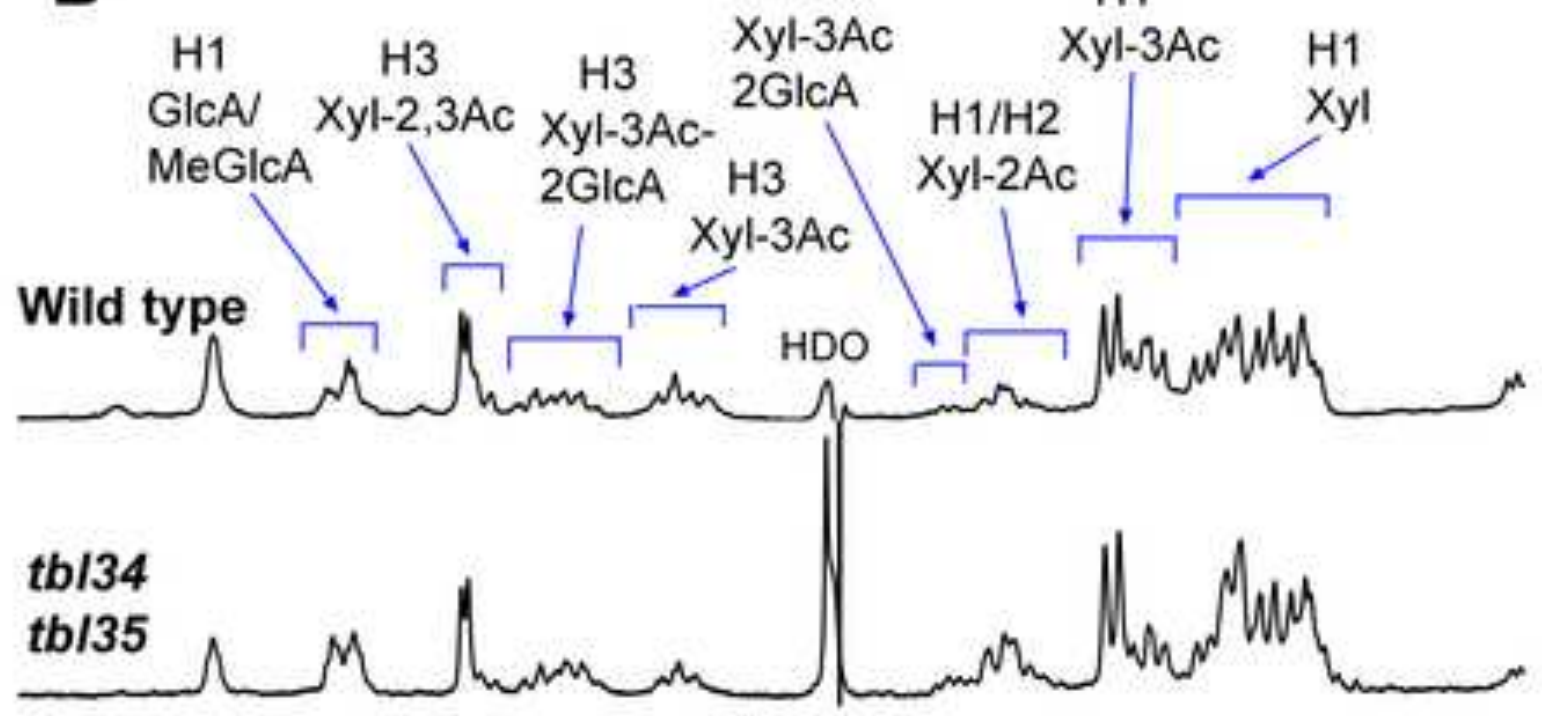

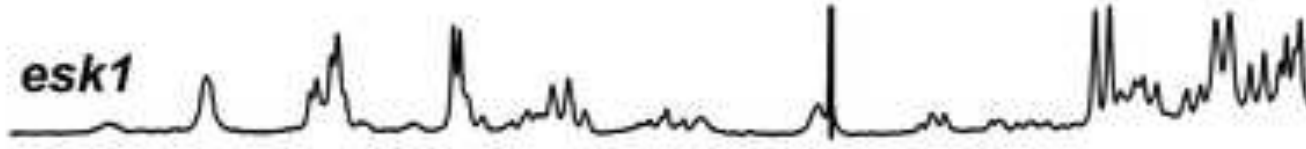
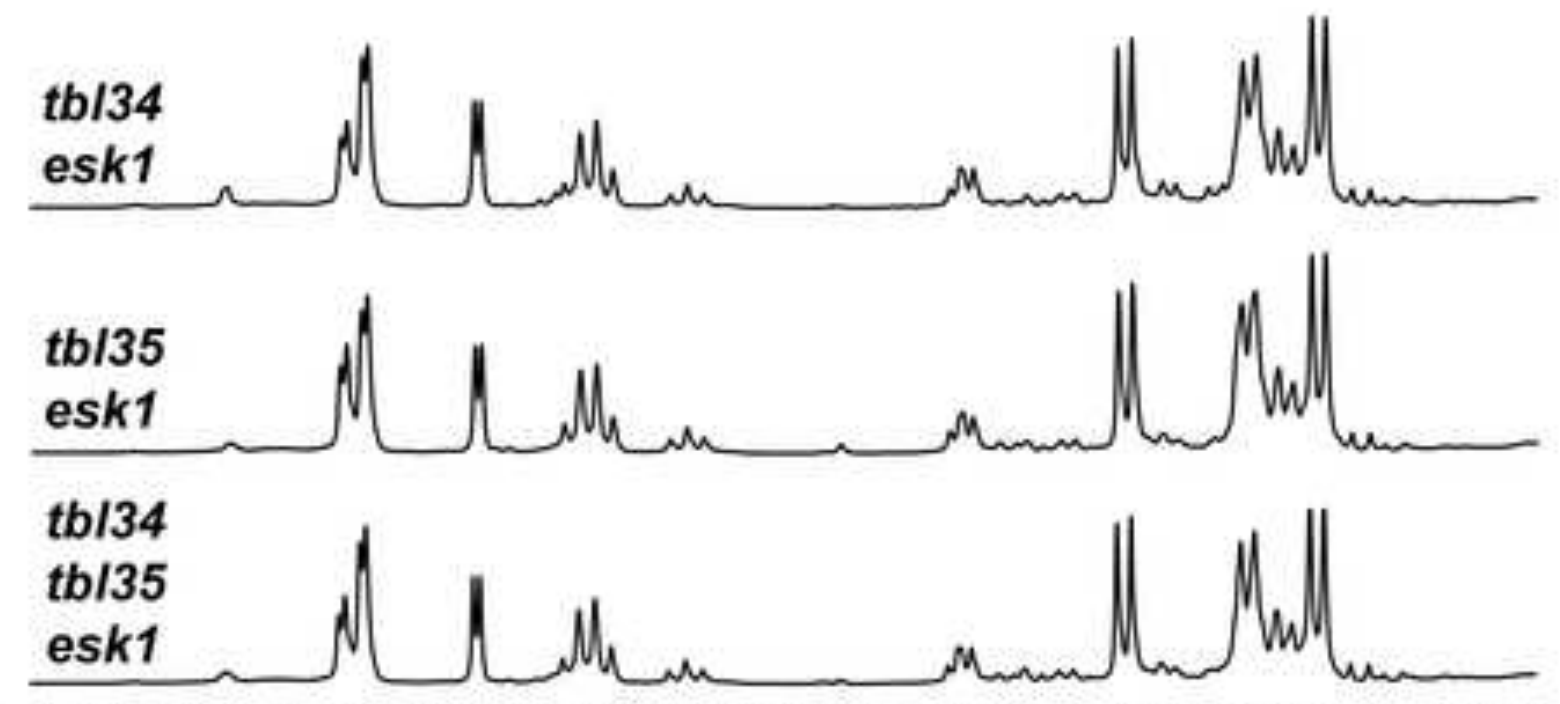

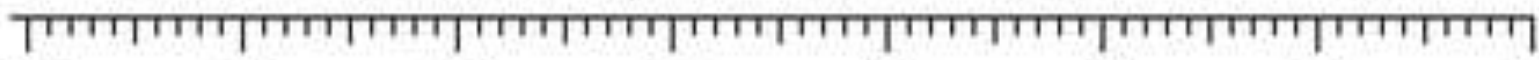

5.6

5.4

5.2

5.0

4.8

4.6

4.4

4.2

\section{${ }^{1} \mathrm{H}$ Chemical Shift (ppm)}

